\title{
Dynamic multi-patch isogeometric analysis of planar Euler-Bernoulli beams
}

\author{
Duy Voa , Aleksandar Borković ${ }^{b, c}$, Pruettha Nanakorn ${ }^{\mathrm{a}}$, Tinh Quoc Bui ${ }^{\mathrm{d}, \mathrm{e}, *}$ \\ ${ }^{a}$ School of Civil Engineering and Technology, Sirindhorn International Institute of Technology, \\ Thammasat University, Pathumthani, Thailand \\ ${ }^{b}$ University of Banja Luka, Faculty of Architecture, Civil Engineering and Geodesy, Department of \\ Mechanics and Theory of Structures, 78000 Banja Luka, Bosnia and Herzegovina \\ 'Institute of Applied Mechanics, Graz University of Technology, Technikerstraße 4/II, 8010 Graz, \\ Austria \\ ${ }^{d}$ Institute for Research and Development, Duy Tan University, Da Nang City, Vietnam \\ ${ }^{e}$ Department of Civil and Environmental Engineering, Tokyo Institute of Technology, 2-12-1-W8-22, \\ Ookayama, Meguro-ku, Tokyo 152-8552, Japan
}

\begin{abstract}
This study presents a novel isogeometric Euler-Bernoulli beam formulation for in-plane dynamic analysis of multi-patch beam structures. The kinematic descriptions involve only displacements of the beam axis, which are approximated by non-uniform rational B-spline (NURBS) curves. Translational displacements of the control points are here considered as control variables. The motivation of this work is to propose a penalty-free method to handle in-plane dynamic analysis of multi-patch beam structures. A simple relation between cross-sectional rotations at the ends of the beams and control variables is derived, allowing the incorporation of the end rotations as degrees of freedom. This improved setting can straightforwardly tackle beam structures with many rigid multi-patch connections, a major challenging issue when using existing isogeometric Euler-Bernoulli beam formulations. Additionally, rotational boundary conditions are conveniently prescribed. Numerical examples with complicated beam structures such as circular arches and frames with kinks are considered to show the accuracy and performance of the developed formulation. The computed results are verified with those derived from the conventional finite element method, and the superior convergence properties of the proposed formulation are illustrated. We additionally discuss about the possible extension of the present approach to spatial beam structures.

Keywords: Isogeometric analysis (IGA); Multi-patch beam structures; Linear dynamic analysis; Superior order of convergence; Circular arches; Frames with kinks;

*Corresponding author.
\end{abstract}


E-mail addresses: duyvo.ce@gmail.com (D. Vo); aleksandar.borkovic@aggf.unibl.org (A. Borković); nanakorn@siit.tu.ac.th (P. Nanakorn); buiquoctinh@duytan.edu.vn \& bui.t.aa@m.titech.ac.jp (T. Q. Bui) 


\section{Introduction}

Isogeometric analysis (IGA) [1] has become an appealing and reliable approach in the field of computational mechanics. The major goal is to achieve a smooth transition from geometric design to analysis using computer-aided design (CAD) basis functions, e.g., non-uniform rational B-spline (NURBS) basis functions, for both processes. In the last decades, IGA has experienced a rapid evolution with many engineering applications, for example, vibration analysis [1, 2], structural analysis [3-8], damage and fracture problems $[9,10]$, optimization $[11,12]$. In particular, dynamic analysis of beam structures using the IGA approach has been studied in recent years. Several Timoshenko beam formulations have been presented in [13-16] for planar, and in [17, 18] for spatial beam structures. Many types of beam are covered, i.e., straight [13], circular, elliptical, parabolic, and free-form beams [14-16], and several material models are involved, i.e., linear elastic, functionally graded materials [16]. Since cross-sectional rotations are considered as degrees of freedom, multi-patch beam structures are straightforwardly analyzed. Results reported in those works reveal the superiority of the IGA approach for vibration of beams with complex geometry. However, using Timoshenko beam formulations for analysis of thin beams requires additional treatments to deal with the well-known shear-locking issue $[17,18]$.

Regarding analysis of thin beams, Euler-Bernoulli beam formulations are more efficient. The shearlocking effect is naturally eliminated in the kinematic assumptions. It is possible to describe structural behavior of thin beams by only translational displacements of the beam axis. Recently, several planar rotation-free isogeometric beam formulations have been developed, e.g., see Refs. [19-26], without the need for considering cross-sectional rotations as discrete unknowns, showing its obvious advantage in reduction of the number of degrees of freedom. In [19], a formulation to optimize elastic arches for the maximum fundamental frequency is presented, and then in [20] nonlinear vibration analysis of straight beams with a von Kármán strain-displacement relation is performed. In [21], higher order mass matrices are constructed based on the consistent counterparts, which aim to improve the order of convergence for frequencies. However, only quadratic and cubic NURBS basis functions are considered, and only straight beams are analyzed. For curved beams, a formulation reported in [22] is for nonlinear dynamic analysis using a linear Biot stress-strain relation. Nonlinear vibration analysis of simply supported free-form curved beams is studied in [23]. Dynamic responses of beam subjected to moving vehicles are elaborated in [24], and effects of curve reparameterization on vibration analysis of free-form curved beams are studied in [25]. Recently, a beam formulation for linear dynamic analysis of an arbitrary curved planar beam is presented in [26], elaborating the influence of the product of the maximum curvature and the cross-section height on the accuracy. 
Although the rotation-free isogeometric beam formulations provide promising results, numerical examples considered involve only beam structures whose geometry can be represented by only one NURBS patch. In fact, avoiding using cross-sectional rotations as degrees of freedom rises several practical difficulties, i.e., consideration of multi-patch beam structures and easy prescriptions of rotational boundary conditions. To the authors' best knowledge, only one rotation-free beam formulation in [27] is applicable to dynamic analysis of multi-patch beam structures. The penalty-like bending strip method [4] is adapted to deal with rigid multi-patch connections. The accuracy of the solution is influenced by an artificial stiffness coefficient, which is found to be problem-dependent. Another important issue is that it is not straightforward to automatically add bending strips to beam structures with many rigid multi-patch connections. As a result, it seems that an efficient isogeometric Euler-Bernoulli beam formulation for dynamic analysis of multi-patch beam structures is still missing.

The objective of the present work is to develop an effective isogeometric Euler-Bernoulli beam formulation particularly dealing with dynamic analysis of planar multi-patch beam structures. In this setting, kinematics are described by using only displacements of the beam axis. NURBS curves are utilized to approximate the beam axis, and displacements of the control points are considered as control variables. Different from previous studies, the cross-sectional rotations at the ends of the beams are incorporated as degrees of freedom in the present formulation, which essentially aims to eliminate the drawbacks of the existing rotation-free isogeometric beam formulations. This is accomplished by presenting a relation between the cross-sectional rotations at the ends and the control variables. The accuracy of this approach has been investigated in [28] with geometrically nonlinear static examples. In this study, this approach is further extended to consider inertia effects, and thus for the first time applied to in-plane dynamic analysis of multi-patch beams.

The rest of the paper is structured as follows. In Section 2, several major aspects are discussed, i.e., kinematic descriptions with definitions of strain measurements and cross-sectional resultant forces, expression of the virtual work principle, discretization of the unknown kinematics using NURBS curves, derivation of the system equations, and incorporation of the end rotations as degrees of freedom for analysis of multi-patch beam structures. In Section 3, several numerical examples with benchmark and complicated structures are considered, in which the convergence, normalized numeric discrete spectra, and transient analysis, are examined to show the accuracy and efficiency of the proposed formulation. In Section 4, the extensibility of the proposed formulation for the analysis of spatial Euler-Bernoulli beams is discussed. Some conclusions drawn from the study are given in Section 5.

\section{Kinematics of a planar curved Euler-Bernoulli beam}




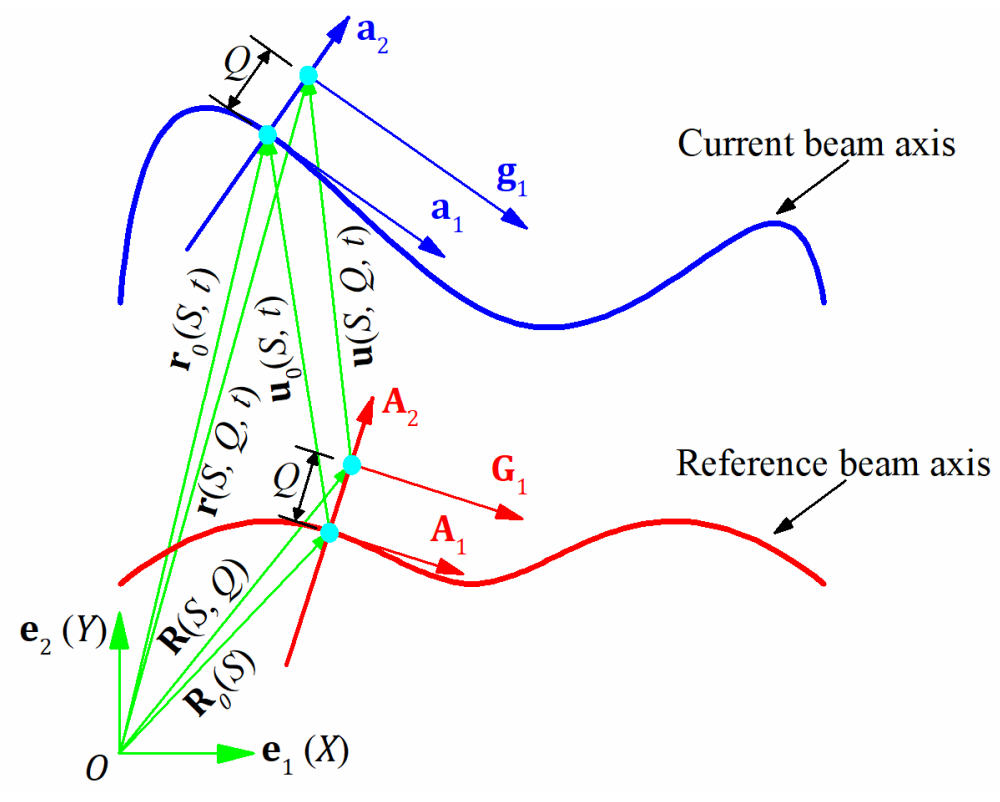

Fig. 1. Kinematic quantities in the reference and current beam configurations.

Beam configurations with kinematic assumptions by means of the Euler-Bernoulli beam theory are schematically sketched in Fig. 1. The unstressed (or initial) configuration is considered as the reference configuration. Any material point of the beam can be determined through a pair of two parametric variables, i.e., $S$ and $Q$, where $S$ is defined as the arc-length variable of the reference beam axis. For notational simplicity, the upper-case letters denote the quantities in the reference configuration while the lower-case letters represent the quantities in the current one.

\subsection{Kinematics}

Position vectors of a point on the beam axis in reference and current configurations are expressed as

$$
\mathbf{R}_{0}(S)=X_{0}(S) \mathbf{e}_{1}+Y_{0}(S) \mathbf{e}_{2} \quad \mathbf{r}_{0}(S, t)=\mathbf{R}_{0}(S)+\mathbf{u}_{0}(S, t)
$$

where $\mathbf{e}_{1}$ and $\mathbf{e}_{2}$ are two base vectors of a global Cartesian coordinate system, $t$ is the time variable, and $\mathbf{u}_{0}(S, t)$ is the displacement vector of the beam axis. The tangent vectors of the beam axis in the reference and current configurations are determined through the following expressions as

$$
\mathbf{A}_{1}=\frac{\mathrm{d} \mathbf{R}_{0}(S)}{\mathrm{d} S}=\mathbf{R}_{0}^{\prime}(S) \quad \mathbf{a}_{1}=\frac{\partial \mathbf{r}_{0}(S, t)}{\partial S}=\mathbf{r}_{0}^{\prime}(S, t)=\mathbf{A}_{1}+\mathbf{u}_{0}^{\prime}(S, t)
$$


Hereafter, a prime denotes the derivative with respect to $S$. It is noted that $\mathbf{A}_{1}$ is a unit vector since $S$ is the arc-length variable of the reference beam axis. Furthermore, with the small displacement assumptions, i.e., $\left\|\mathbf{u}_{0}(S, t)\right\| \ll 1$ and $\left\|\mathbf{u}_{0}^{\prime}(S, t)\right\| \ll 1$, the following condition can be reached:s

$$
\left\|\mathbf{a}_{1}\right\| \approx\left\|\mathbf{A}_{1}\right\|=1
$$

The normal vectors of the beam axis in the reference and current configurations are calculated as

$$
\mathrm{A}_{2}=\Lambda \mathrm{A}_{1}
$$

where $\boldsymbol{\Lambda}$ is a rotation tensor given by

$$
\Lambda=\left[\begin{array}{cc}
\cos \frac{\pi}{2} & -\sin \frac{\pi}{2} \\
\sin \frac{\pi}{2} & \cos \frac{\pi}{2}
\end{array}\right]=\left[\begin{array}{cc}
0 & -1 \\
1 & 0
\end{array}\right]
$$

Now the position vectors of an arbitrary point of the beam in both the reference and current configurations are written as

$$
\mathbf{R}(S, Q)=\mathbf{R}_{0}(S)+Q \mathbf{A}_{2} \quad \mathbf{r}(S, Q, t)=\mathbf{r}_{0}(S, t)+Q \mathbf{a}_{2}
$$

and consequently, the displacement vector of an arbitrary point is computed as

$$
\mathbf{u}(S, Q, t)=\mathbf{r}(S, Q, t)-\mathbf{R}(S, Q)=\mathbf{u}_{0}(S, t)+Q \mathbf{\Lambda} \mathbf{u}_{0}^{\prime}(S, t)
$$

\subsection{Strain measurements and cross-sectional resultant forces}

In terms of the Green-Lagrange strain tensor, E, the only non-zero strain component at an arbitrary point of the beam continuum, i.e., $E_{11}$, is expressed as [28]

$$
E_{11}=\overbrace{\frac{1}{2}\left(\mathbf{a}_{1} \cdot \mathbf{a}_{1}-\mathbf{A}_{1} \cdot \mathbf{A}_{1}\right)}^{\Gamma_{11}}+Q \overbrace{\left(\mathbf{A}_{2} \cdot \mathbf{A}_{1}^{\prime}-\mathbf{a}_{2} \cdot \mathbf{a}_{1}^{\prime}\right)}^{K_{11}}
$$

with $\Gamma_{11}$ and $K_{11}$ being the axial strain and curvature change of the beam axis, respectively. Substituting Eqs. (2) and (4) into Eq. (8), and neglecting the higher order terms of $\mathbf{u}_{0}(S)$ and its derivatives, one can derive the following strain measurements as 


$$
\Gamma_{11}=\mathbf{A}_{1} \cdot \mathbf{u}_{0}^{\prime}(S, t) \quad K_{11}=-\mathbf{A}_{2}^{\prime} \cdot \mathbf{u}_{0}^{\prime}(S, t)-\mathbf{A}_{2} \cdot \mathbf{u}_{0}^{\prime \prime}(S, t)
$$

It is important to notice that these strain measurements can be considered as the linearized versions of the counterparts derived in [28].

The second Piola-Kirchhoff stress tensor $\mathbf{S}$ is used as the energy conjugate of the Green-Lagrange strain tensor $\mathbf{E}$. Since $\mathbf{E}$ has only one non-zero component, it follows that

$$
S^{11}=E E_{11}
$$

where $E$ is Young's modulus. Cross-sectional resultant forces, i.e., normal force $N^{11}$ and bending moment $M^{11}$, are defined as the integrals of $S^{11}$ and its moment over the cross-section as

$$
N^{11}=\int_{A} S^{11} \mathrm{~d} A=E A \Gamma_{11} \quad M^{11}=\int_{A} S^{11} Q \mathrm{~d} A=E I K_{11}
$$

with $A$ and $I$ being the cross-sectional area and moment of inertia, respectively. The generalized strain vector $\boldsymbol{\Gamma}$ and stress vector $\mathbf{N}$ are then defined as

$$
\boldsymbol{\Gamma}=\left[\begin{array}{ll}
\Gamma_{11} & K_{11}
\end{array}\right]^{\mathrm{T}} \quad \mathbf{N}=\left[\begin{array}{ll}
N^{11} & M^{11}
\end{array}\right]^{\mathrm{T}} .
$$

Using Eq. (11) yields

$$
\mathbf{N}=\left[\begin{array}{cc}
E A & 0 \\
0 & E I
\end{array}\right] \boldsymbol{\Gamma}=\mathbf{D} \boldsymbol{\Gamma} .
$$

In subsequent subsections, equations are given in matrix forms for computational benefits.

\subsection{Virtual work principle}

The virtual work principle consists of three terms, i.e.,

$$
\delta \Pi_{\text {iner }}+\delta \Pi_{i n t}-\delta \Pi_{\text {ext }}=0
$$

where $\delta \Pi_{\text {iner }}$ is the inertial virtual work, $\delta \Pi_{\text {int }}$ is the internal virtual work, and $\delta \Pi_{\text {ext }}$ is the external virtual work. They are expressed as 


$$
\begin{aligned}
& \delta \Pi_{\text {iner }}=\int_{V} \delta \mathbf{u}^{\mathrm{T}} \rho \ddot{\mathbf{u}} \mathrm{d} V=\int_{0}^{L}\left[\delta \mathbf{u}_{0}^{\mathrm{T}} \rho A \ddot{\mathbf{u}}_{0}+\left(\delta \mathbf{u}_{0}^{\prime}\right)^{\mathrm{T}} \rho I \ddot{\mathbf{u}}_{0}^{\prime}\right] \mathrm{d} S \\
& \delta \Pi_{\text {int }}=\int_{V} \delta E_{11} S^{11} \mathrm{~d} V=\int_{0}^{L} \delta \boldsymbol{\Gamma}^{\mathrm{T}} \mathbf{N} \mathrm{d} S \quad \delta \Pi_{\text {ext }}=\int_{0}^{L} \delta \mathbf{u}_{0}^{\mathrm{T}} \mathbf{f} \mathrm{d} S+\delta \mathbf{u}_{0}^{\mathrm{T}}(0) \mathbf{p}(0)+\delta \mathbf{u}_{0}^{\mathrm{T}}(L) \mathbf{p}(L) .
\end{aligned}
$$

In the above expressions, $V$ and $L$ are the volume and length of the reference beam configuration, and $\rho$ is the mass density. Additionally, $\mathbf{f}$ is the distributed force vector applied on the beam axis while $\mathbf{p}(0)$ and $\mathbf{p}(L)$ are the concentrated forces applied at $S=0$ and $S=L$, respectively. Furthermore, a double dot notation denotes the second derivative with respect to time, i.e.,

$$
(\ddot{*})=\frac{\partial^{2}(*)}{\partial t^{2}} \text {. }
$$

\subsection{NURBS discretization and system equations}

The non-uniform rational B-spline (NURBS) curves are used for the present analysis, and we decided to omit its introduction. Curious readers may refer to, e.g., [29] for details. The position vectors of the beam axis in the reference and current configurations are approximated by NURBS curves as

$$
\mathbf{R}_{0}(\xi)=\sum_{i=1}^{n} R_{i, p}(\xi) \mathbf{R}_{i} \quad \mathbf{r}_{0}(\xi, t)=\sum_{i=1}^{n} R_{i, p}(\xi) \mathbf{r}_{i}(t)
$$

where $R_{i, p}(\xi)$ are rational B-spline basis functions of degree $p, \xi$ is the parametric variable referred to as the knot variable, and $n$ is the number of basis functions. In addition, $\mathbf{R}_{i}$ and $\mathbf{r}_{i}(t)$ are the position vectors of the $i$ th control point in the reference and current configurations, respectively. For simple notations, the degree $p$ is omitted since now on.

Kinematics of the beam axis are discretized with the same functions as the beam axis as

$$
\begin{aligned}
& \mathbf{u}_{0}(\xi, t)=\mathbf{r}_{0}(\xi, t)-\mathbf{R}_{0}(\xi)=\sum_{i=1}^{n} R_{i}(\xi)\left(\mathbf{r}_{i}-\mathbf{R}_{i}\right)=\sum_{i=1}^{n} R_{i}(\xi) \mathbf{u}_{i}(t) \\
& \mathbf{u}_{0}^{\prime}(\xi, t)=\sum_{i=1}^{n} R_{i}^{\prime}(\xi) \mathbf{u}_{i}(t) \quad \mathbf{u}_{0}^{\prime \prime}(\xi, t)=\sum_{i=1}^{n} R_{i}^{\prime \prime}(\xi) \mathbf{u}_{i}(t) \quad \ddot{\mathbf{u}}_{0}(\xi, t)=\sum_{i=1}^{n} R_{i}(\xi) \ddot{\mathbf{u}}_{i}(t)
\end{aligned}
$$


where $\mathbf{u}_{i}(t)$ and $\ddot{\mathbf{u}}_{i}(t)$ are the displacement and acceleration vectors of the $i$ th control point, respectively.

For later compact expressions of the system equations, some matrices are introduced as follows:

$$
\begin{array}{llll}
\mathbf{c}=\left[\begin{array}{lllll}
\mathbf{u}_{1}^{\mathrm{T}} & \mathbf{u}_{2}^{\mathrm{T}} & \ldots & \mathbf{u}_{n}^{\mathrm{T}}
\end{array}\right]^{\mathrm{T}} & \ddot{\mathbf{c}}=\left[\begin{array}{lllll}
\ddot{\mathbf{u}}_{1}^{\mathrm{T}} & \ddot{\mathbf{u}}_{2}^{\mathrm{T}} & \ldots & \ddot{\mathbf{u}}_{n}^{\mathrm{T}}
\end{array}\right]^{\mathrm{T}} \\
\mathbf{H}=\left[\begin{array}{lllllll}
R_{1}(\xi) \mathbf{I} & R_{2}(\xi) \mathbf{I} & \ldots & R_{n}(\xi) \mathbf{I}
\end{array}\right] & \mathbf{H}^{\prime}=\left[\begin{array}{lllll}
R_{1}^{\prime}(\xi) \mathbf{I} & R_{2}^{\prime}(\xi) \mathbf{I} & \ldots & R_{n}^{\prime}(\xi) \mathbf{I}
\end{array}\right]
\end{array}
$$

where $\mathbf{I}$ is an identity matrix of size $2 \times 2$. Here the vector $\mathbf{c}$ is referred to as the vector of control variables. From Eqs. (19) and (20), it follows that

$$
\mathbf{u}_{0}(\xi, t)=\mathbf{H c} \quad \mathbf{u}_{0}^{\prime}(\xi, t)=\mathbf{H}^{\prime} \mathbf{c} \quad \ddot{\mathbf{u}}_{0}(\xi, t)=\mathbf{H} \ddot{\mathbf{c}} .
$$

Recall the generalized strain vector $\boldsymbol{\Gamma}$ in Eq. (12), its discretization is expressed as

$$
\boldsymbol{\Gamma}=\sum_{i=1}^{n} \mathbf{B}_{i} \mathbf{u}_{i}=\mathbf{B c}
$$

where the matrices $\mathbf{B}_{i}$ and $\mathbf{B}$ are given by

$$
\mathbf{B}_{i}=\left[\begin{array}{c}
R_{i}^{\prime}(\xi) \mathbf{A}_{1}^{\mathrm{T}} \\
-R_{i}^{\prime}(\xi)\left(\mathbf{A}_{2}^{\prime}\right)^{\mathrm{T}}-R_{i}^{\prime \prime}(\xi) \mathbf{A}_{2}^{\mathrm{T}}
\end{array}\right] \quad \quad \mathbf{B}=\left[\begin{array}{llll}
\mathbf{B}_{1} & \mathbf{B}_{2} & \ldots & \mathbf{B}_{n}
\end{array}\right]
$$

A point load vector $\mathbf{P}$ is introduced as

$$
\mathbf{P}=\left[\begin{array}{lllll}
\mathbf{p}^{\mathrm{T}}(0) & \mathbf{0}^{\mathrm{T}} & \ldots & \mathbf{0}^{\mathrm{T}} & \mathbf{p}^{\mathrm{T}}(L)
\end{array}\right]^{\mathrm{T}}
$$

with $\mathbf{0}$ being a vector of zeros of size $2 \times 1$.

Substituting Eqs. (23), (24), and (26) into Eqs. (15) and (16) yields

$$
\begin{aligned}
& \delta \Pi_{\text {iner }}=\delta \mathbf{c}^{\mathrm{T}}\left[\int_{0}^{L}\left(\rho A \mathbf{H}^{\mathrm{T}} \mathbf{H}+\rho I\left(\mathbf{H}^{\prime}\right)^{\mathrm{T}} \mathbf{H}^{\prime}\right) \mathrm{d} S\right] \ddot{\mathbf{c}}=\delta \mathbf{c}^{\mathrm{T}} \mathbf{M} \ddot{\mathbf{c}} \\
& \delta \Pi_{i n t}=\delta \mathbf{c}^{\mathrm{T}}\left(\int_{0}^{L} \mathbf{B}^{\mathrm{T}} \mathbf{D B} \mathrm{d} S\right) \mathbf{c}=\delta \mathbf{c}^{\mathrm{T}} \mathbf{K} \mathbf{c} \quad \delta \Pi_{\text {ext }}=\delta \mathbf{c}^{\mathrm{T}} \int_{0}^{L} \mathbf{H}^{\mathrm{T}} \mathbf{f} \mathrm{d} S+\delta \mathbf{c}^{\mathrm{T}} \mathbf{P}=\delta \mathbf{c}^{\mathrm{T}} \mathbf{F} .
\end{aligned}
$$

The discrete equations in terms of control variables of an undamped, forced beam are obtained by substituting Eqs. (27) and (28) into Eq. (14) as 


$$
\mathbf{M} \ddot{\mathbf{c}}+\mathbf{K c}=\mathbf{F} .
$$

Here, $\mathbf{M}, \mathbf{K}$, and $\mathbf{F}$ are the mass matrix, stiffness matrix, and force vector, respectively.

It is important to point out here that only the displacements of the beam axis are unknown kinematics, and the displacements of control points, i.e., the vector of control variables $\mathbf{c}$, are considered as discrete unknowns. To analyze beams with rigid multi-patch connections, the end rotations of the beams should be incorporated as degrees of freedom, and, for that purpose, a transformation between control variables and these rotations is required. It will be presented subsequently.

\subsection{Connections of multiple beams with the end rotations}

The NURBS approximation of the reference beam axis is given in Fig. 2a. Relations between the displacements of the second and second-to-last control points (i.e., $\mathbf{R}_{2}$ and $\left.\mathbf{R}_{n-1}\right)$ and the end rotations $\left(\theta_{1}\right.$ and $\theta_{n}$ ) can be derived as

$$
\begin{aligned}
& \mathbf{u}_{2}(t)=\mathbf{u}_{1}(t)+L_{1} \theta_{1}(t) \boldsymbol{\Lambda} \mathbf{T}_{1}+\Delta L_{1} \mathbf{T}_{1} \\
& \mathbf{u}_{n-1}(t)=\mathbf{u}_{n}(t)-L_{n-1} \theta_{n}(t) \boldsymbol{\Lambda} \mathbf{T}_{n}-\Delta L_{n-1} \mathbf{T}_{n}
\end{aligned}
$$

where $L_{1}$ and $L_{n-1}$ are the lengths of the first and last segments of the control polygon as shown in Fig. 2a while $\mathbf{T}_{1}$ and $\mathbf{T}_{n}$ are the unit vectors at the two ends of the beam. Moreover, $\Delta L_{1}$ and $\Delta L_{n-1}$ are the changes in length of the first and last segments of the control polygon. It is also noted that the end rotations, i.e., $\theta_{1}(t)$ and $\theta_{n}(t)$, are positive if they are measured in the counterclockwise direction.

Detailed derivations of the relation in Eq. (30) are given here. Fig. $2 \mathrm{~b}$ shows the movements of the first two control points, i.e., from $\mathbf{R}_{1}$ to $\mathbf{r}_{1}$ and from $\mathbf{R}_{2}$ to $\mathbf{r}_{2}$. From the point $\mathbf{r}_{1}$, a line directed by the vector $\mathbf{T}_{1}$ is plotted, and the projection of the point $\mathbf{r}_{2}$ on this line is denoted by $\mathbf{b}$. The vector pointing from $\mathbf{r}_{1}$ to $\mathbf{b}$, i.e., $\mathbf{b}-\mathbf{r}_{1}$, is determined as

$$
\mathbf{b}-\mathbf{r}_{1}=\left(L_{1}+\Delta L_{1}\right) \cos \theta_{1} \mathbf{T}_{1}
$$

The small displacement assumption implies that $\left|\theta_{1}\right| \ll 1$. Consequently, the vector $\mathbf{b}-\mathbf{r}_{1}$ can be expressed as

$$
\mathbf{b}-\mathbf{r}_{1}=\left(L_{1}+\Delta L_{1}\right) \mathbf{T}_{1}=L_{1} \mathbf{T}_{1}+\Delta L_{1} \mathbf{T}_{1}
$$

In addition, the vector pointing from $\mathbf{b}$ to $\mathbf{r}_{2}$ is determined as 


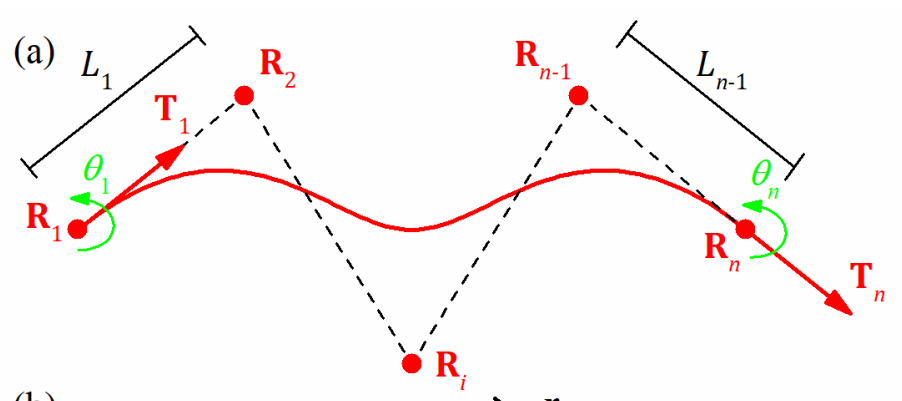

(b)

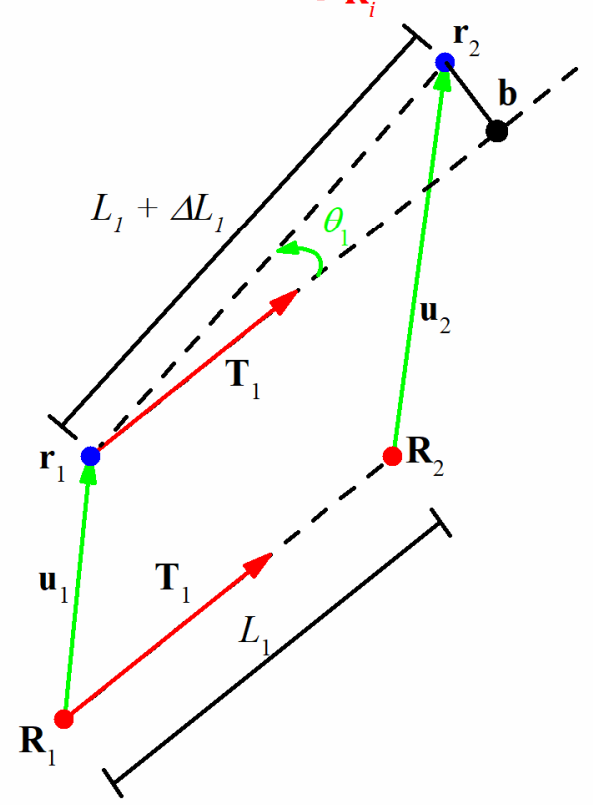

Fig. 2. (a) NURBS approximation of the reference beam axis and the end rotations. (b) Movements of the first two control points.

$\mathbf{r}_{2}-\mathbf{b}=\left(L_{1}+\Delta L_{1}\right) \sin \theta_{1} \boldsymbol{\Lambda} \mathbf{T}_{1}=L_{1} \theta_{1} \boldsymbol{\Lambda} \mathbf{T}_{1}$

Here again, $\sin \theta_{1} \approx \theta_{1}$ and $\left|\Delta L_{1} \theta_{1}\right| \ll 1$ due to the small displacement assumption.

By referring to Fig. 2b, the following relation is obtained:

$$
\mathbf{u}_{1}+\mathbf{r}_{2}-\mathbf{r}_{1}=\mathbf{u}_{2}+\mathbf{R}_{2}-\mathbf{R}_{1}
$$

or

$$
\begin{aligned}
\mathbf{u}_{2} & =\mathbf{u}_{1}+\mathbf{r}_{2}-\mathbf{r}_{1}+\mathbf{R}_{2}-\mathbf{R}_{1} \\
& =\mathbf{u}_{1}+\mathbf{r}_{2}-\mathbf{b}+\mathbf{b}-\mathbf{r}_{1}+\mathbf{R}_{2}-\mathbf{R}_{1}
\end{aligned}
$$




$$
\begin{aligned}
& =\mathbf{u}_{1}+L_{1} \theta_{1} \boldsymbol{\Lambda} \mathbf{T}_{1}+L_{1} \mathbf{T}_{1}+\Delta L_{1} \mathbf{T}_{1}-L_{1} \mathbf{T}_{1} \\
& =\mathbf{u}_{1}+L_{1} \theta_{1} \boldsymbol{\Lambda} \mathbf{T}_{1}+\Delta L_{1} \mathbf{T}_{1}
\end{aligned}
$$

The relation in Eq. (31) can be obtained in a similar manner.

A new vector of degrees of freedom $\mathbf{d}$ is defined as

$$
\begin{aligned}
\mathbf{d} & =\left[\begin{array}{lllllllll}
\mathbf{u}_{1}^{\mathrm{T}} & \theta_{1} & \Delta L_{1} & \mathbf{u}_{3}^{\mathrm{T}} & \ldots & \mathbf{u}_{n-2}^{\mathrm{T}} & \Delta L_{n-1} & \mathbf{u}_{n}^{\mathrm{T}} & \theta_{n}
\end{array}\right]^{\mathrm{T}} \\
\ddot{\mathbf{d}} & =\left[\begin{array}{lllllllll}
\ddot{\mathbf{u}}_{1}^{\mathrm{T}} & \ddot{\theta}_{1} & \Delta \ddot{L}_{1} & \ddot{\mathbf{u}}_{3}^{\mathrm{T}} & \ldots & \ddot{\mathbf{u}}_{n-2}^{\mathrm{T}} & \Delta \ddot{L}_{n-1} & \ddot{\mathbf{u}}_{n}^{\mathrm{T}} & \ddot{\theta}_{n}
\end{array}\right]^{\mathrm{T}} .
\end{aligned}
$$

The transformation between the vectors $\mathbf{c}$ and $\mathbf{d}$, with the use of Eqs. (30) and (31), can be obtained as

$$
\mathbf{c}=\mathbf{T d} \quad \ddot{\mathbf{c}}=\mathbf{T} \ddot{\mathbf{d}} .
$$

A new point load vector $\mathbf{P}_{\mathbf{d}}$ is introduced as

$$
\mathbf{P}_{\mathbf{d}}=\left[\begin{array}{lllll}
\mathbf{p}^{\mathrm{T}}(0) & m_{1} & \cdots & \mathbf{p}^{\mathrm{T}}(L) & m_{n}
\end{array}\right]^{\mathrm{T}}
$$

where $m_{1}$ and $m_{n}$ are concentrated moments at the first and last ends of the beam, respectively.

Substituting Eqs. (39) and (40) into Eqs. (27) and (28) yields

$$
\begin{aligned}
& \delta \Pi_{\text {iner }}=\delta \mathbf{d} \mathbf{T}^{\mathrm{T}} \mathbf{M} \mathbf{T} \ddot{\mathbf{d}}=\delta \mathbf{d} \mathbf{M}_{\mathbf{d}} \ddot{\mathbf{d}} \\
& \delta \Pi_{i n t}=\delta \mathbf{d} \mathbf{T}^{\mathrm{T}} \mathbf{K} \mathbf{T} \mathbf{d}=\delta \mathbf{d} \mathbf{K}_{\mathbf{d}} \mathbf{d} \quad \delta \Pi_{\text {ext }}=\delta \mathbf{d}^{\mathrm{T}} \mathbf{T}^{\mathrm{T}} \int_{0}^{L} \mathbf{H}^{\mathrm{T}} \mathbf{f} \mathrm{d} S+\delta \mathbf{d}^{\mathrm{T}} \mathbf{P}_{\mathbf{d}}=\delta \mathbf{d}^{\mathrm{T}} \mathbf{F}_{\mathbf{d}} .
\end{aligned}
$$

The discrete equations in terms of degrees of freedom for an undamped, forced beam are

$$
\mathbf{M}_{\mathbf{d}} \ddot{\mathbf{d}}+\mathbf{K}_{\mathbf{d}} \mathbf{d}=\mathbf{F}_{\mathbf{d}}
$$

\subsection{Modal and transient analysis}

For modal analysis, a synchronous and harmonic solution is assumed as

$$
\mathbf{d}=\overline{\mathbf{d}} e^{k \omega t}
$$

where $k^{2}=-1$. This assumption reduces the homogeneous part to a generalized eigenproblem as 


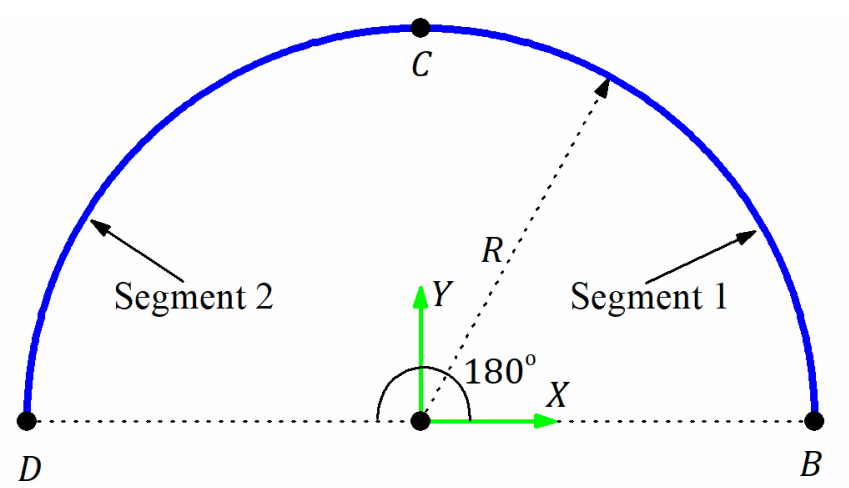

Fig. 3. Semi-circular arch - Problem setup.

(a)

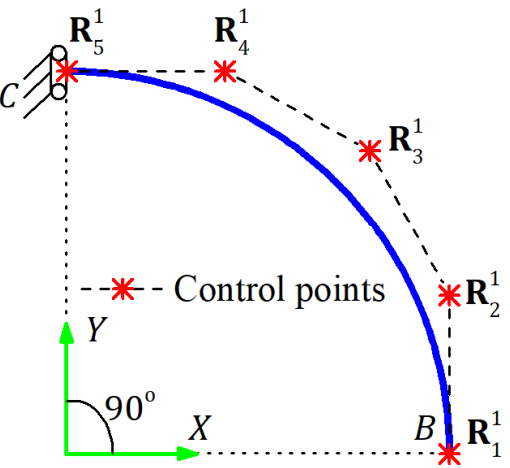

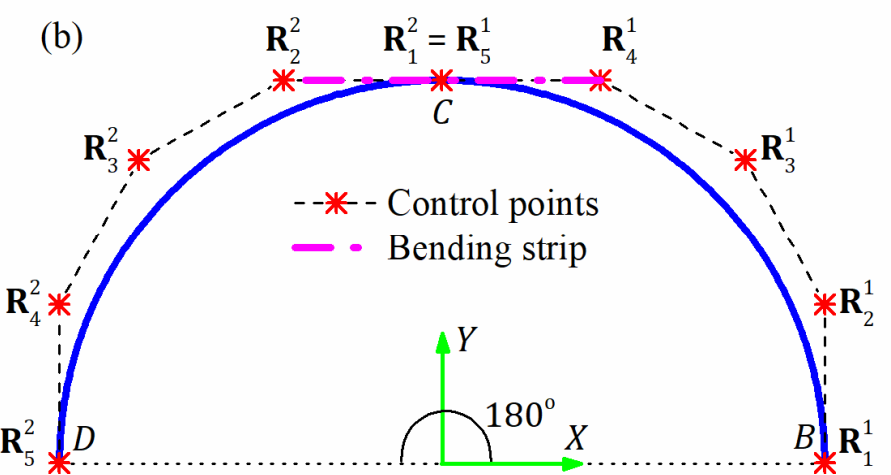

Fig. 4. Semi-circular arch. (a) Equivalent model for static analysis with symmetric boundary conditions. (b) Model for vibration analysis using a bending strip.

$$
\left(\mathbf{K}_{\mathbf{d}}-\omega^{2} \mathbf{M}_{\mathbf{d}}\right) \overline{\mathbf{d}}=\mathbf{0}
$$

where $\omega$ is a natural frequency, and $\overline{\mathbf{d}}$ is a normalized natural vibration mode shape. The widely-used normalization scheme is also employed here, i.e.,

$$
(\overline{\mathbf{d}})^{\mathbf{T}} \mathbf{M}_{\mathbf{d}} \overline{\mathbf{d}}=1 .
$$

The transient analysis is performed using the Newmark method with constant-average acceleration.

\section{Numerical examples and discussions}

In this section, several numerical examples are considered to show the accuracy and performance of the proposed formulation. For comparison, results obtained by the present approach are compared with those obtained by the conventional finite element approach using classical two-noded Euler-Bernoulli beam elements with the following interpolation schemes, i.e., the linear interpolations of the axial displacement 
Table 1. Semi-circular arch. Comparison of the first five normalized natural frequencies $\bar{\omega}_{j}=$ $\omega_{j} R^{2} \sqrt{\rho A / E I}$.

\begin{tabular}{|c|c|c|c|c|}
\hline \multirow{2}{*}{ Mode } & \multicolumn{2}{|c|}{ Pinned-pinned } & \multicolumn{2}{|c|}{ Fixed-fixed } \\
\hline & $2 \mathrm{nEB}$ & Present & $2 \mathrm{nEB}$ & Present \\
\hline 1 & 2.267 & 2.267 & 4.384 & 4.384 \\
\hline 2 & 6.923 & 6.922 & 9.650 & 9.65 \\
\hline 3 & 13.976 & 13.975 & 17.919 & 17.918 \\
\hline 4 & 22.815 & 22.813 & 27.513 & 27.511 \\
\hline 5 & 33.922 & 33.919 & 39.782 & 39.780 \\
\hline
\end{tabular}

and the curvature, and the cubic polynomial interpolation of the transverse displacement. In fact, this beam element is widely considered in commercial finite element packages, e.g., beam element B52 in MarcMentat and B23 in Abaqus. In this study, this beam element is referred to as the 2nEB element.

The convergence tests are performed for the natural frequencies. The converged results in each approach are produced by using a large number of degrees of freedom (DOFs), namely 2397 DOFs for the semi-circular arch and the L-shaped frame, 2388 DOFs for the circular ring, and 2307 DOFs for the lattice tower. Additionally, sextic NURBS curves with maximum inter-element continuity are used for the converged solutions of the proposed formulation.

\subsection{A semi-circular arch}

The first example is devoted to analysis of a semi-circular arch of radius $R$ shown in Fig. 3. The arch consists of two segments, i.e., $B C$ and $C D$. Since each segment is a quadrant, NURBS representations of the segments are straightforwardly constructed. Two different symmetric boundary conditions are prescribed at $B$ and $D$ as pinned-pinned and fixed-fixed, respectively. Some characteristics of the proposed formulation, i.e., the convergence properties, accuracy of high frequencies per DOFs, and transient behavior, are investigated.

Major drawbacks of rotation-free isogeometric beam formulations for the static analysis of the arch are briefly discussed. Fig. 4a shows an equivalent model for the case of symmetric loading and boundary conditions. Due to the lack of rotational DOFs, the rotational boundary condition at $C$ is treated by 
(a)
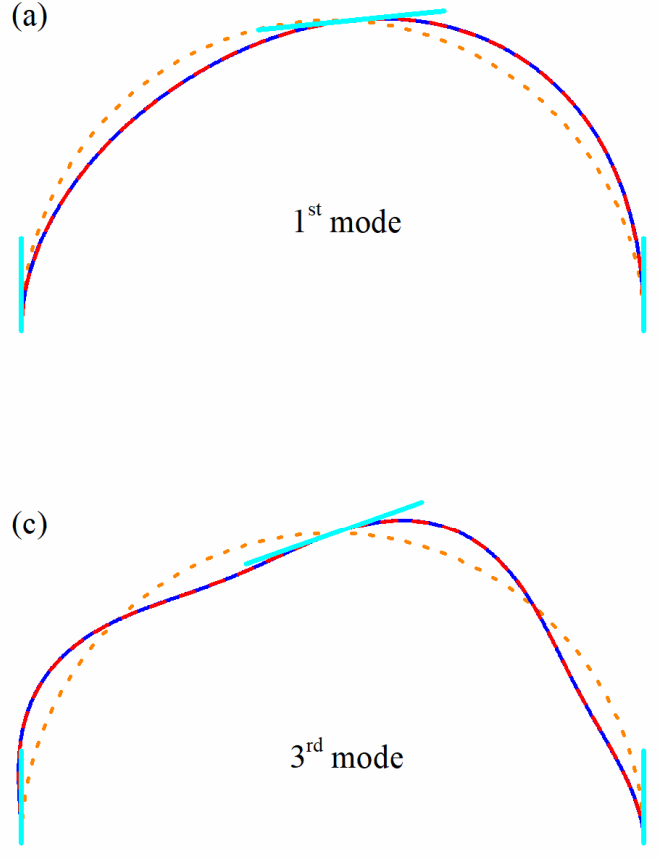

(b)

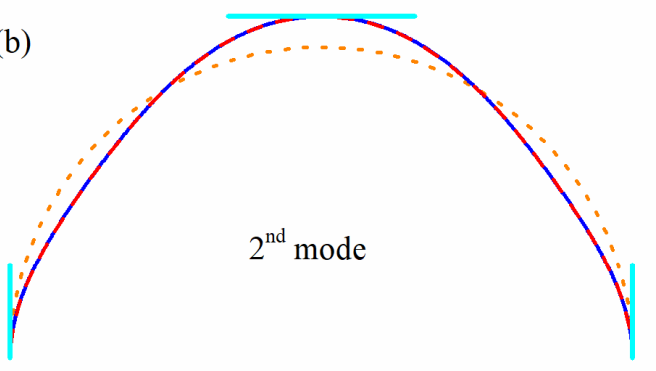

(d)

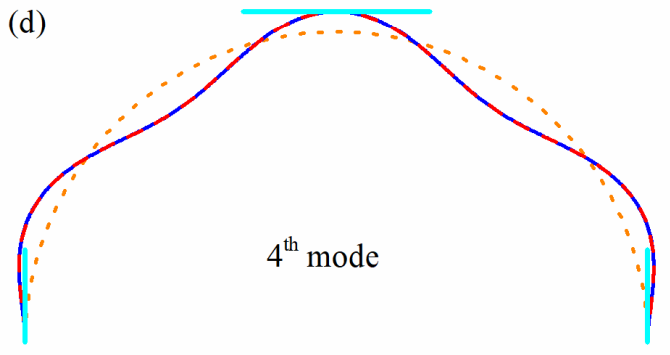

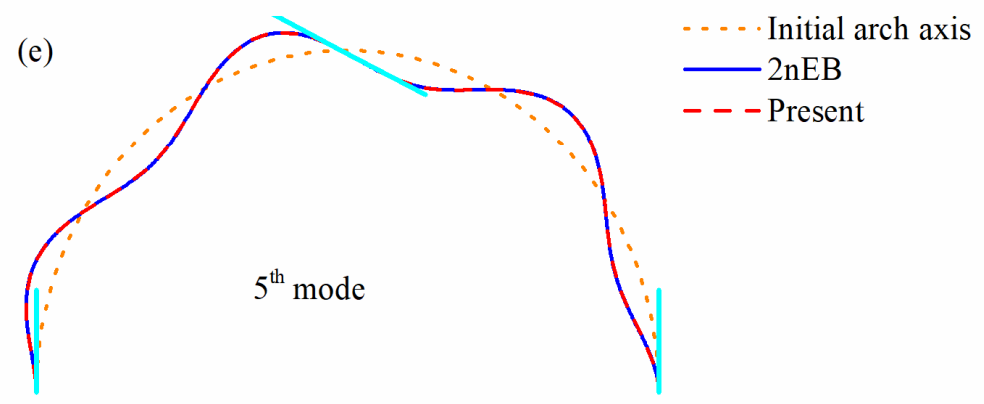

Fig. 5. Semi-circular arch - First five mode shapes with fixed-fixed boundary conditions.

constraining the vertical displacements of the control points $\mathbf{R}_{4}^{1}$ and $\mathbf{R}_{5}^{1}$ to be equal. Basically, the requirement of additional constraints in analysis of beam structures under several rotational boundary conditions is critical as determinations of all the constraints may not be a trivial task.

For the vibration analysis, regardless of the symmetric loading and boundary conditions, the whole arch must be simulated due to the presence of asymmetric vibration modes. Using rotation-free isogeometric beam formulations, the rigid multi-patch connection at $C$ can be imposed using a bending strip as shown in Fig. 4b. However, the rigid multi-patch connection at $C$ and rotational boundary conditions at $B$ and $D$ are straightforwardly and automatically treated with the developed formulation since the rotational DOFs are included, reflecting one of the advantages of the present approach. 


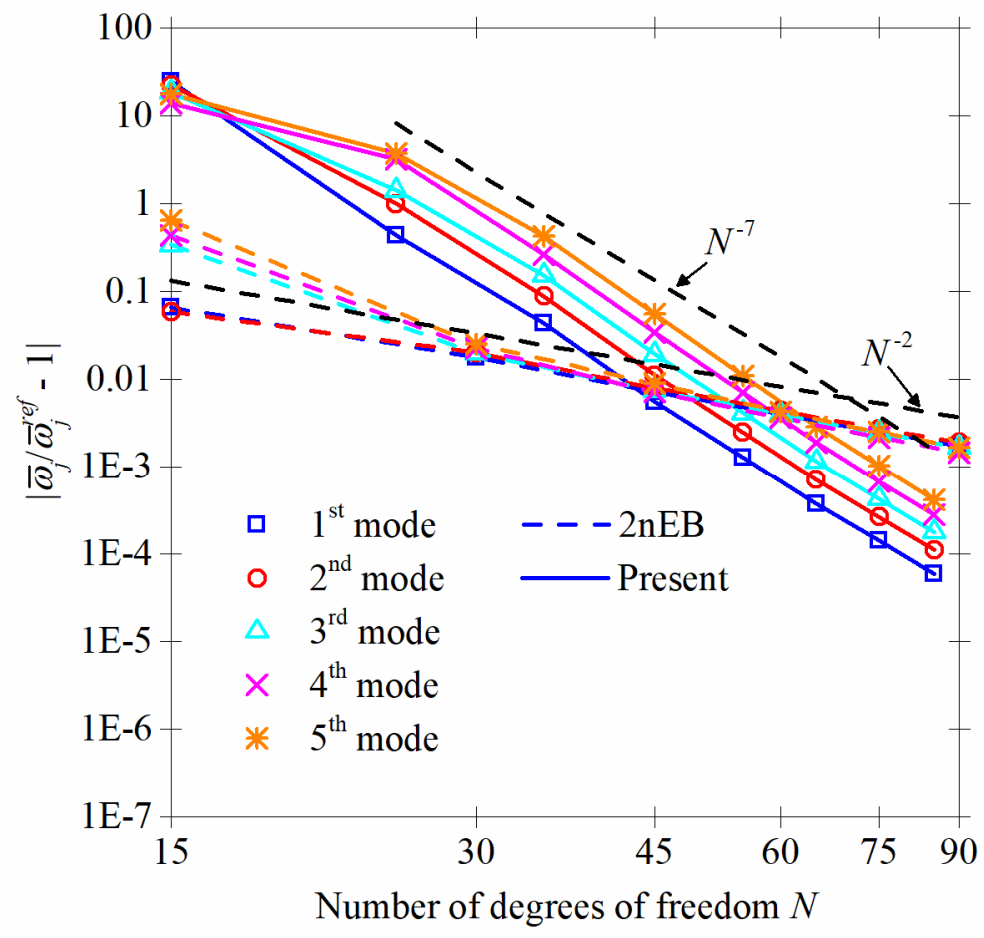

Fig. 6. Semi-circular arch - Convergence test for the first five $\bar{\omega}_{j}$ between the $2 \mathrm{nEB}$ and the proposed formulation with cubic NURBS curves.

\subsubsection{Accuracy verification}

In this example, a natural frequency of the arch is normalized by

$$
\bar{\omega}_{j}=\omega_{j} R^{2} \sqrt{\frac{\rho A}{E I}} .
$$

Results of the first five $\bar{\omega}_{j}$ obtained by both the $2 \mathrm{nEB}$ element and the proposed formulation are reported in Table 1, revealing very good agreement. The first five mode shapes of the arch with the fixed-fixed boundary conditions are plotted in Fig. 5. Identical mode shapes are obtained by the 2nEB element and the present approach. It is emphasized that three asymmetric mode shapes are found, i.e., $1^{\text {st }}, 3^{r d}$, and $5^{\text {th }}$ mode shapes.

The enforcement of the rigid multi-patch connection at $C$ and rotational boundary conditions at $B$ and $D$ is illustrated. The tangent vectors of the arch at $B, C$ and $D$ are visualized as extended lines in Fig. 5. The tangent vectors in the vertical direction at the fixed supports $B$ and $D$ imply no rotations. Regarding the 

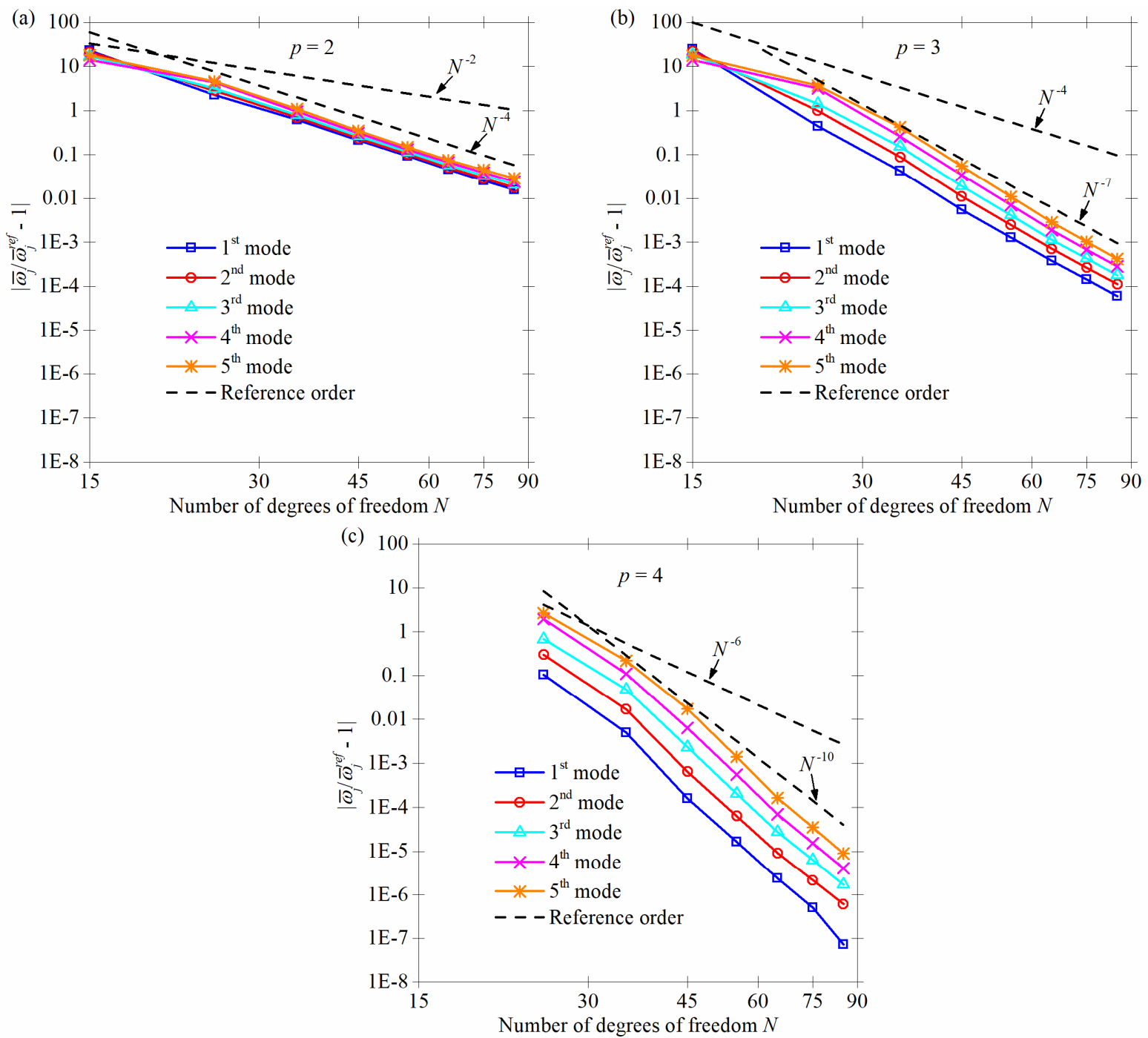

Fig. 7. Semi-circular arch - Convergence test for different NURBS degree $p$. (a) $p=2$. (b) $p=3$. (c)

$$
p=4 \text {. }
$$

rigid multi-patch connection at $C$, the tangent vectors are determined separately from two segments $B C$ and $C D$. Their alignment illustrates the perfect enforcement of the multi-patch connection at $C$.

\subsubsection{Efficiency verification}

Convergence tests are performed with the relative errors of the first five $\bar{\omega}_{j}$. The relative errors are considered as functions of the number of DOFs $N$. Since the 2nEB element uses the cubic polynomial for the interpolation of the transverse displacement, results obtained using cubic NURBS curves are used for comparison. Computed results for the relative errors are plotted in Fig. 6. It can be observed that the higher 

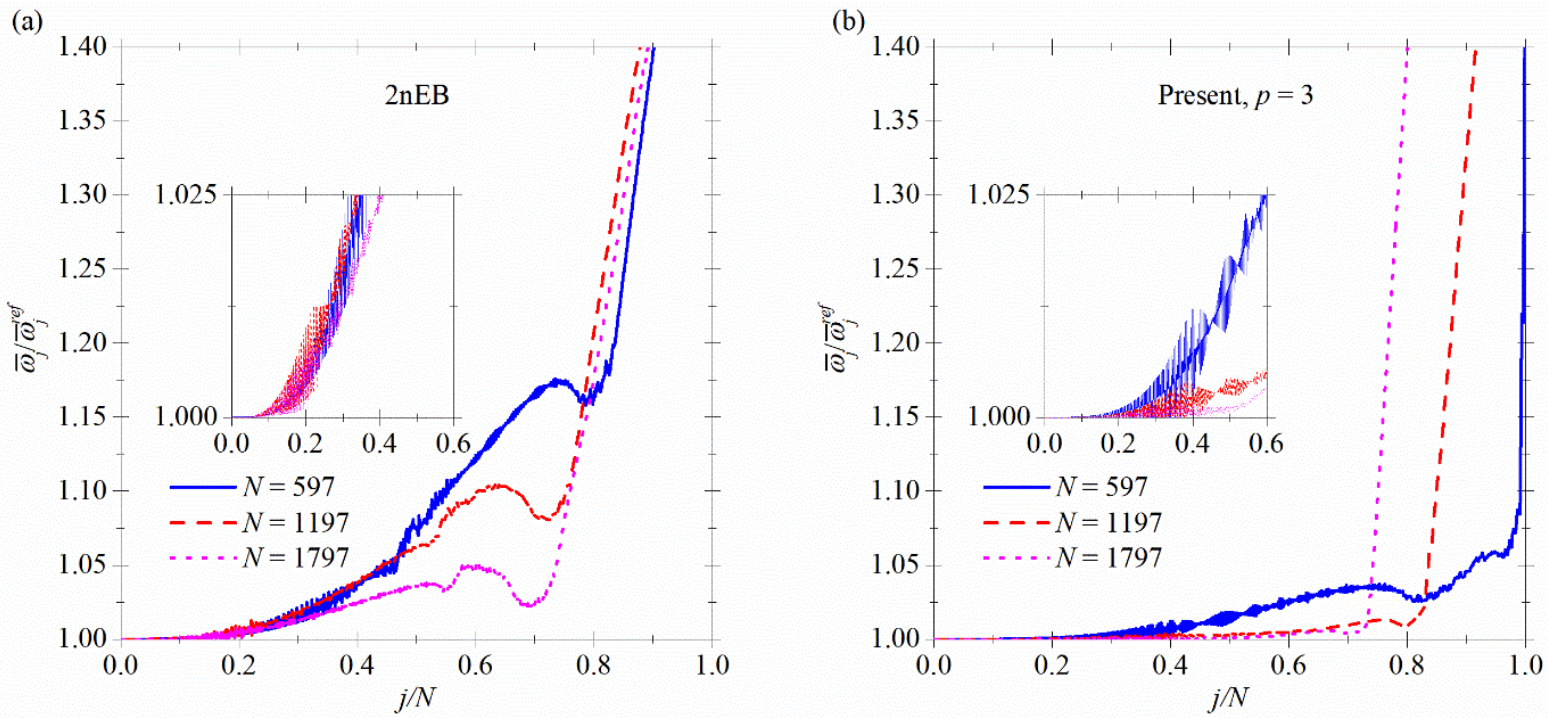

Fig. 8. Semi-circular arch - Normalized numeric discrete spectra. (a) the $2 \mathrm{nEB}$ formulation. (b) The proposed formulation.

order of convergence is obtained with the proposed formulation. Indeed, the orders of convergence of the $2 \mathrm{nEB}$ element and the proposed formulation are, respectively, equal to 2 and 7.

\subsubsection{Convergence analysis}

In this experiment, the relative errors of the first five $\bar{\omega}_{j}$ are still considered as functions of the number of DOFs $N$. The arch is analyzed using several NURBS curves of different degree $p$, i.e., $p=2$, 3 , and 4 . Computed results for the relative errors are shown in Fig. 7. The accuracy increases significantly with the use of NURBS curves of higher degree. In addition, the accuracy of lower frequencies, e.g., $\bar{\omega}_{1}$, is better than that of higher ones, e.g., $\bar{\omega}_{5}$. Regarding the order of convergence, as studied in $[1,26]$, results obtained with the use of NURBS curves of degree $p$ are expected to converge with the order of $2(p-1)$. In this example, it is found that the present formulation exhibits superior convergence properties. In fact, results of relative errors converge with the order of $3 p-2$, which is higher than $2(p-1)$.

\subsubsection{Normalized numeric discrete spectra}

Normalized numeric discrete spectra are computed for the vibration analysis of the arch. In this experiment, the obtained frequencies $\bar{\omega}_{j}$ are normalized with the converged solutions $\bar{\omega}_{j}^{r e f}$, and then plotted versus the normalized number of DOFs $j / N$. The normalized numeric discrete spectra for the $2 \mathrm{nEB}$ element 

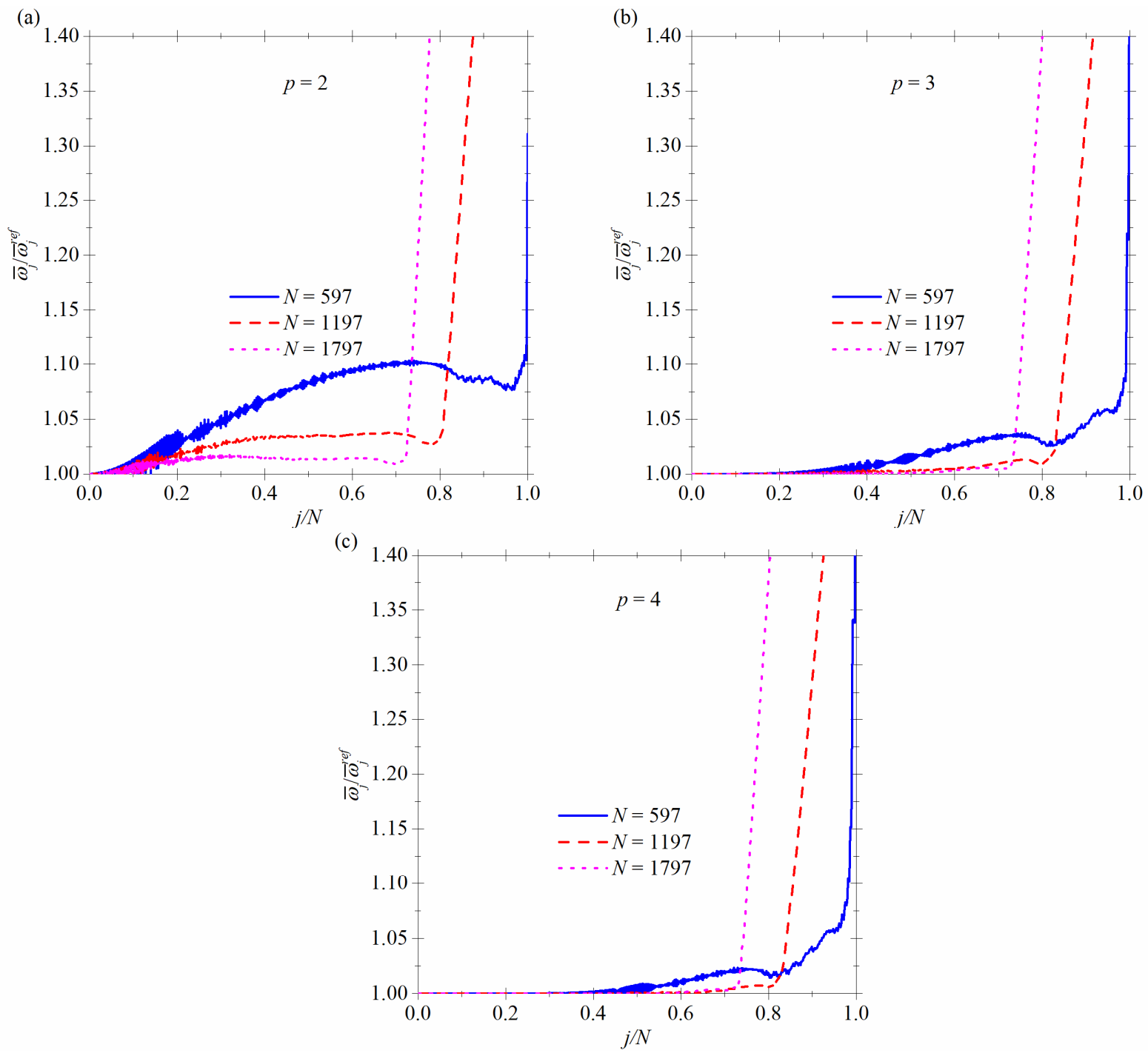

Fig. 9. Semi-circular arch - Normalized numeric discrete spectra with different NURBS degree $p$. (a)

$$
p=2 \text {. (b) } p=3 \text {. (c) } p=4 \text {. }
$$

and the present approach using cubic NURBS curves are shown in Fig. 8a-b. Three different values of $N$, i.e., $N=597,1197$, and 1797, are considered. The number of DOFs $N$ greatly alters the spectra. This observation is consistent with the similar experiment performed in [26]. For all values of $N$ considered, results get progressively worse when the ratio $j / N$ reaches a certain value. This behavior is attributed to outlier frequencies which are described in [1]. In the subsequent discussions, this phenomenon is referred to as outlier phenomenon. Although higher accuracy can be obtained, larger values of $N$ seem to lead to earlier occurrences of the outlier phenomenon. For high frequencies, the present approach shows better performance than the conventional finite element approach with the $2 \mathrm{nEB}$ element. Up to $60 \%$ of 


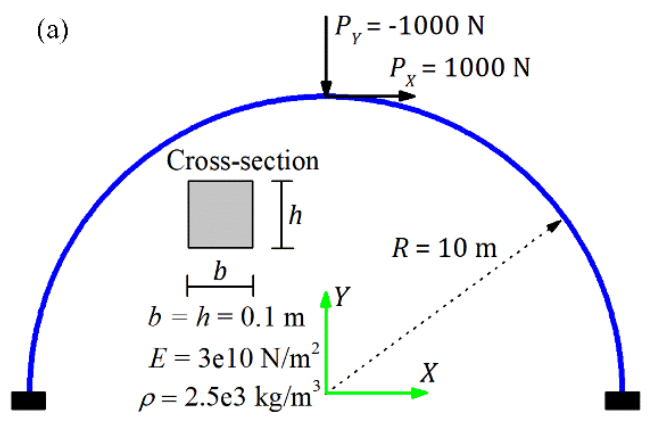

(b)

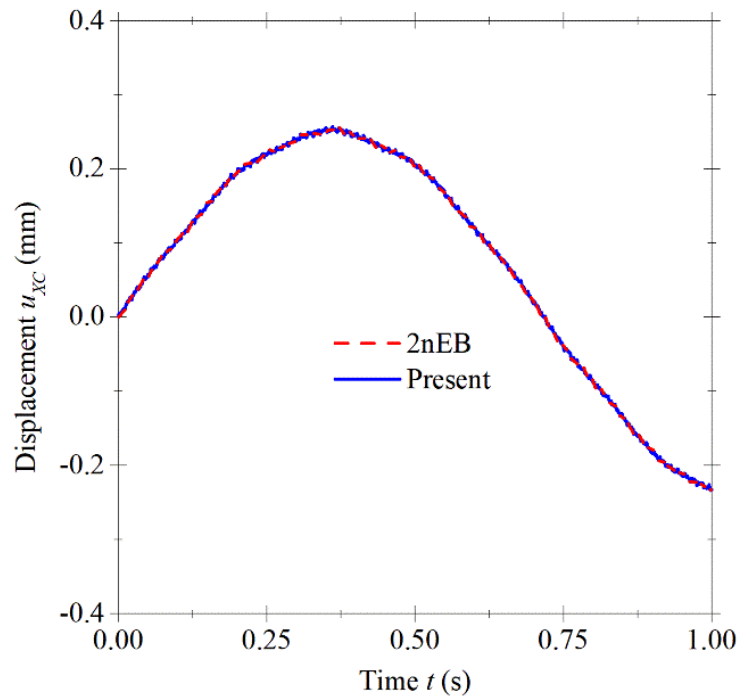

(c)

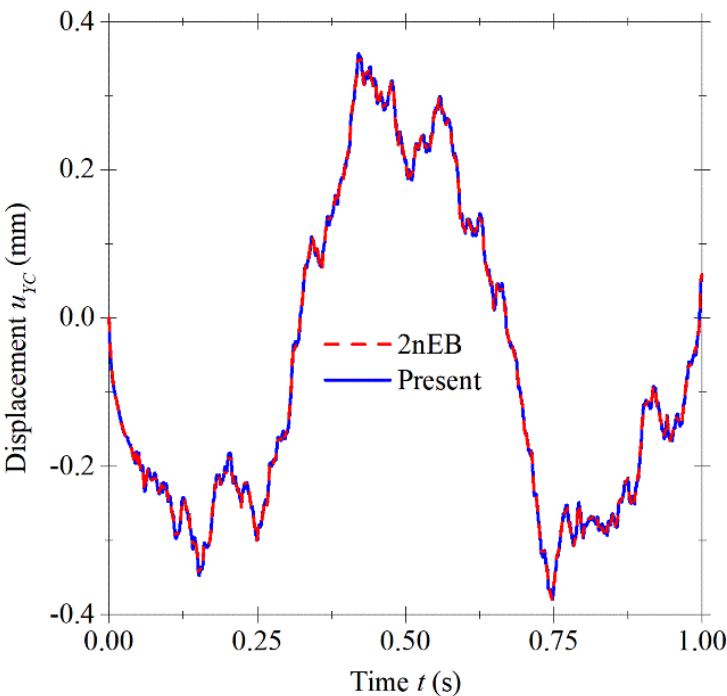

Fig. 10. Semi-circular arch - Transient analysis. (a) Horizontal displacement $u_{X}$. (b) Vertical displacement $u_{Y}$.

frequencies, obtained by the proposed formulation, and only $40 \%$ of frequencies, obtained by the $2 \mathrm{nEB}$ element, have the relative error less than 2.5\%. This finding is depicted in magnified figures in Fig. 8a-b.

Fig. 9 shows the normalized numeric discrete spectra for the several NURBS curves of different degree $p$, i.e., $p=2,3$, and 4 . The accuracy of high frequencies is enhanced by using NURBS curves of higher degree. Once again, the dependency of the spectra on $N$ and the earlier appearances of the outlier phenomenon for higher values of $N$ are detected. Additionally, with the same specified value of $N$, the outlier phenomenon occurs at the almost identical ratios $j / N$ for all the degree $p$.

\subsubsection{Transient analysis}

The problem setup is depicted in Fig. 10a. Here, geometric and material properties are selected as $R=$ $10 \mathrm{~m}, b=h=0.1 \mathrm{~m}, E=3 e 10 \mathrm{~N} / \mathrm{m}^{2}, \rho=2.5 e 3 \mathrm{~kg} / \mathrm{m}^{3}$, where $b$ and $h$ are the cross-section width and 


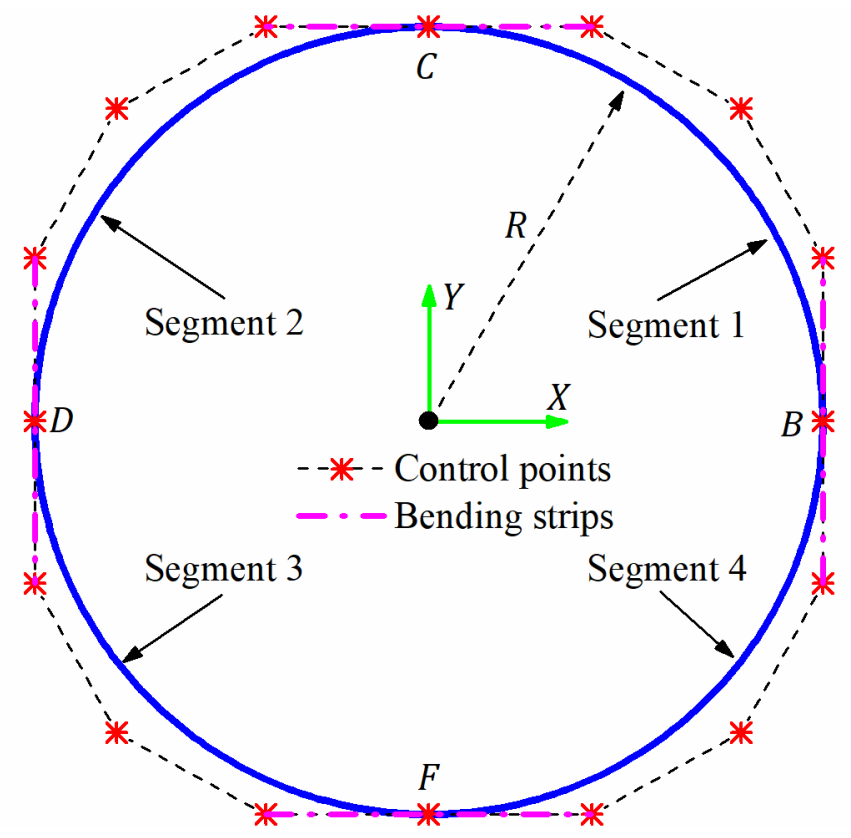

Fig. 11. Circular ring - Problem setup with bending strips.
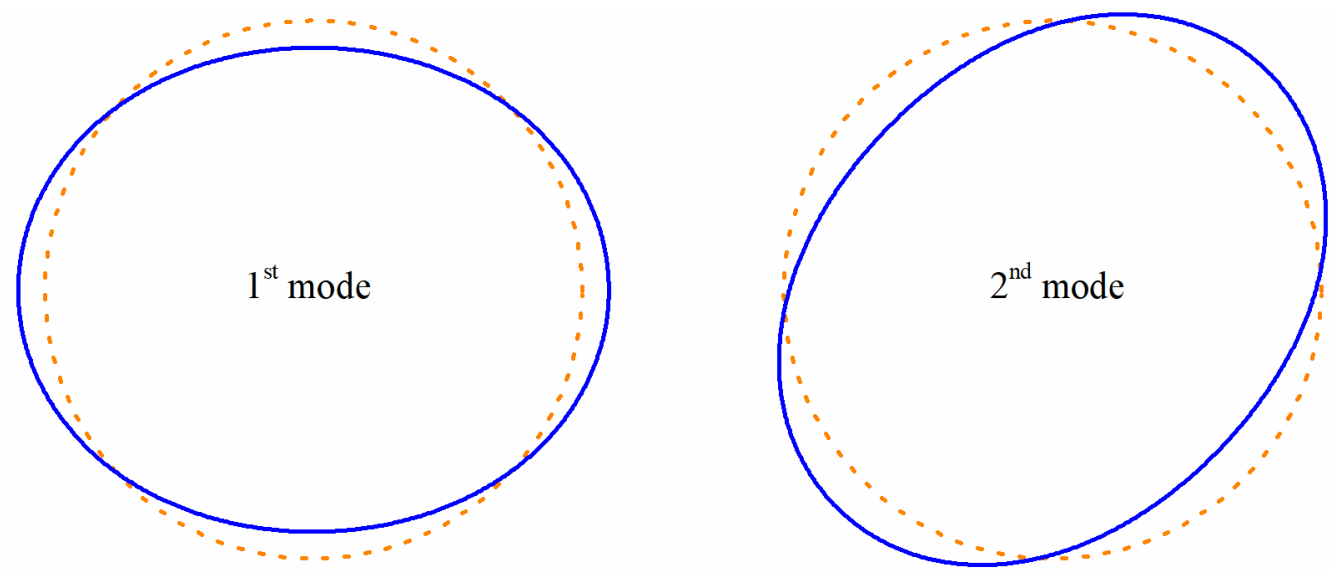

- Initial ring axis

Mode shapes

Fig. 12. Circular ring - The first two mode shapes.

height. A pair of concentrated impulsive forces is applied at $C$, i.e., $P_{X}=1000 \mathrm{~N}$ and $P_{Y}=-1000 \mathrm{~N}$. Fixed-fixed boundary conditions at $B$ and $D$ are considered. With the prescribed loading and boundary conditions, the whole arch is analyzed. To solve discrete equations of motion, the Newmark method is used with the time step $\Delta t=0.0005 \mathrm{~s}$. Additionally, concentrated forces are impulsively applied with a duration $t_{i m}=0.0005 \mathrm{~s}$. Here, the impulsive period $t_{i m}$ and the time step $\Delta t$ are selected to be close to one percent of the period of the mode 10 in order to excite high modes in the transient response of the arch and ensure smooth time histories of displacements, respectively. 
Table 2. Circular arch. Comparison of the five lowest normalized natural frequencies $\bar{\omega}_{j}=$

\begin{tabular}{lll}
\multicolumn{3}{c}{$\omega_{j} R^{2} \sqrt{\rho A / E I}}$. \\
\hline Mode & $2 \mathrm{nEB}$ & Present \\
\hline 1 & 2.683 & 2.683 \\
3 & 7.589 & 7.589 \\
5 & 14.551 & 14.550 \\
7 & & \\
& & \\
9 & 34.519 & 34.517
\end{tabular}

Fig. 10b-c show time histories of the displacements at $C$, i.e., the horizontal displacement $u_{X C}$ and the vertical displacement $u_{Y C}$, obtained by the $2 \mathrm{nEB}$ element and the proposed formulation. In this experiment, 597 DOFs are adequate to obtain the accurate results. Two quartic NURBS curves are employed. Both solutions are identical, which confirms the high accuracy of the present approach in terms of the transient response of the semi-circular arch.

\subsection{A circular ring}

The previous example of a semi-circular arch shows high order of convergence of the natural frequencies obtained by the proposed formulation. This convergence property is further elaborated by considering a full circular ring depicted in Fig. 11. The ring is split into four segments, i.e., $B C, C D, D F$, and $F B$, and no boundary conditions are prescribed. The natural frequency of the ring is normalized as in Eq. (47). If a rotation-free isogeometric beam formulation is employed, four bending strips as shown in Fig. 11 may be used to tackle rigid multi-patch connections at $B, C, D$, and $F$.Such a special treatment using bending strips is no longer required in the present approach.

Since no boundary conditions are prescribed, the first three frequencies are thus equal to zero and their corresponding mode shapes represent rigid-body motions. As a result, the first frequency, i.e., $\bar{\omega}_{1}$, here refers to the first non-zero one. In addition, it is found that $\bar{\omega}_{j}$ and $\bar{\omega}_{j+1}$, where $j$ is an odd number, always have the same value and their mode shapes are identical under a rotation with a certain angle. This phenomenon is depicted in Fig. 12 for the first and second mode shapes. The first mode shape can be simply 

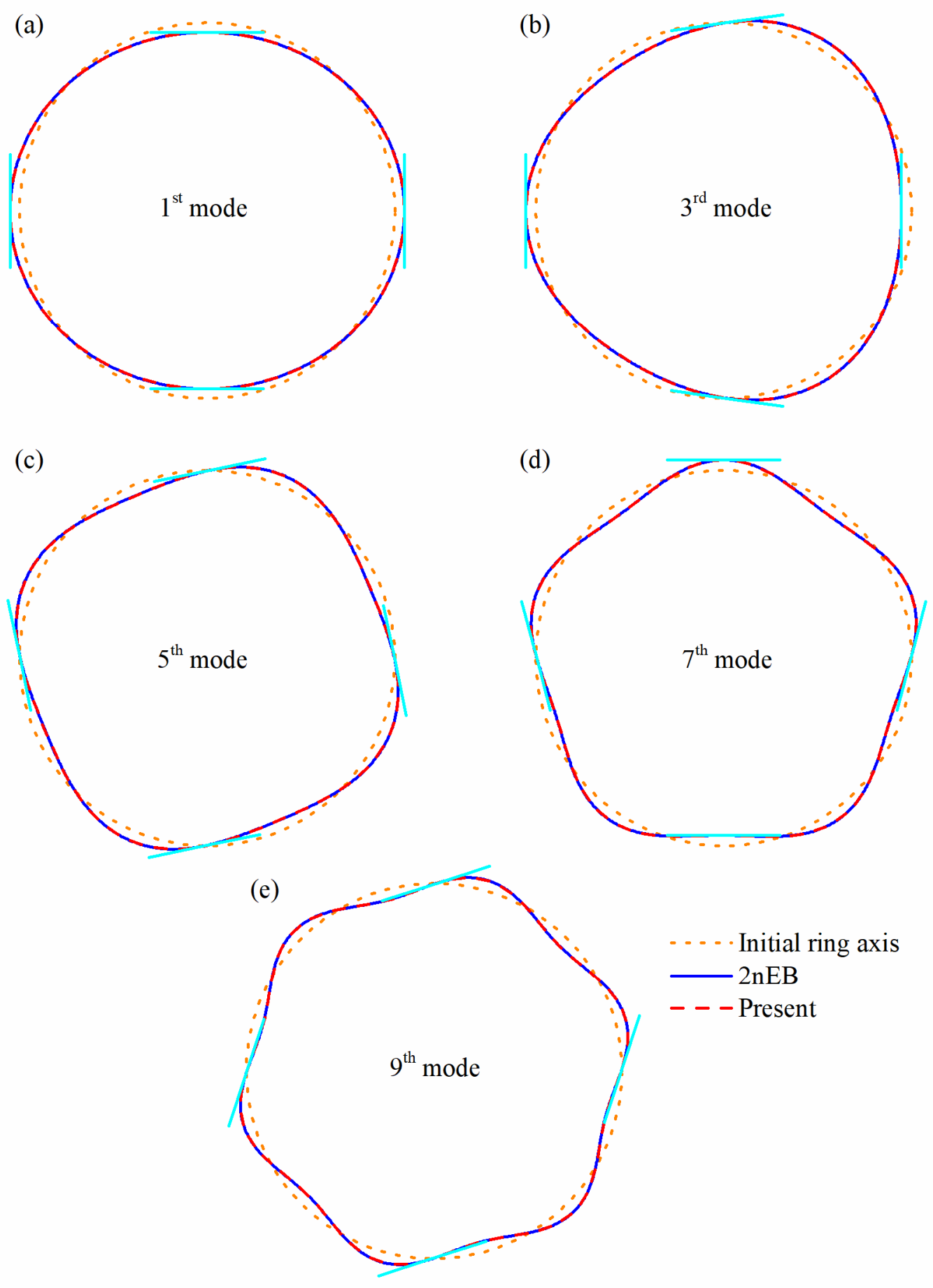

Fig. 13. Circular ring - First five distinct mode shapes.

transformed into the second mode shape by a rotation of 45 degrees in the counterclockwise direction. For comparison, only the distinct frequencies and corresponding mode shapes are therefore considered. 


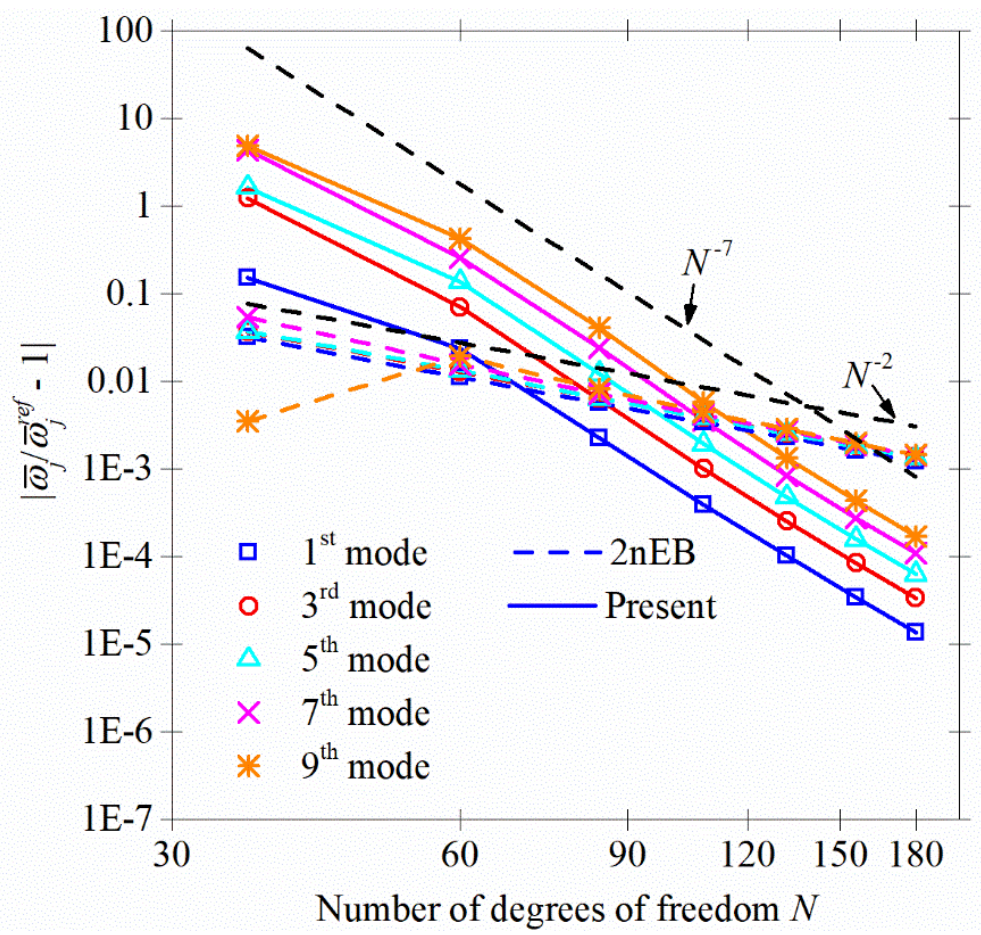

Fig. 14. Circular ring - Convergence test for first five distinct $\bar{\omega}_{j}$ between the $2 \mathrm{nEB}$ element and the proposed formulation with cubic NURBS curves.

The first five distinct frequencies, obtained by the $2 \mathrm{nEB}$ element and the present approach, and their mode shapes are reported in Table 2 and Fig. 13. As expected, there is no much difference between two solutions. In the mode shapes, the extended lines represent the tangent vectors at the points $B, C, D$, and $F$. It is noted that the tangent vectors at each point are determined separately from the two segments connected at the point. The alignments of the tangent vectors imply the perfect enforcement of the rigid multi-patch connections.

The convergence in terms of relative errors for the first five distinct frequencies obtained by both the $2 \mathrm{nEB}$ element and the proposed formulation is visualized in Fig. 14. Cubic NURBS curves are used for result comparison. The figure shows a similar conclusion to that stated in the previous example, i.e., the present approach offers better order of convergence in comparison with the $2 \mathrm{nEB}$ element. Once again, the orders of convergence for the $2 \mathrm{nEB}$ element and the present approach are, respectively, equal to 2 and 7 . Additionally, we also show in Fig. 15 the effects of the degree $p$, i.e., $p=2$, 3, and 4, on the convergence. Once again, the results obtained by the proposed formulation converge with the order of $3 p-2$ instead of $2(p-1)$. 

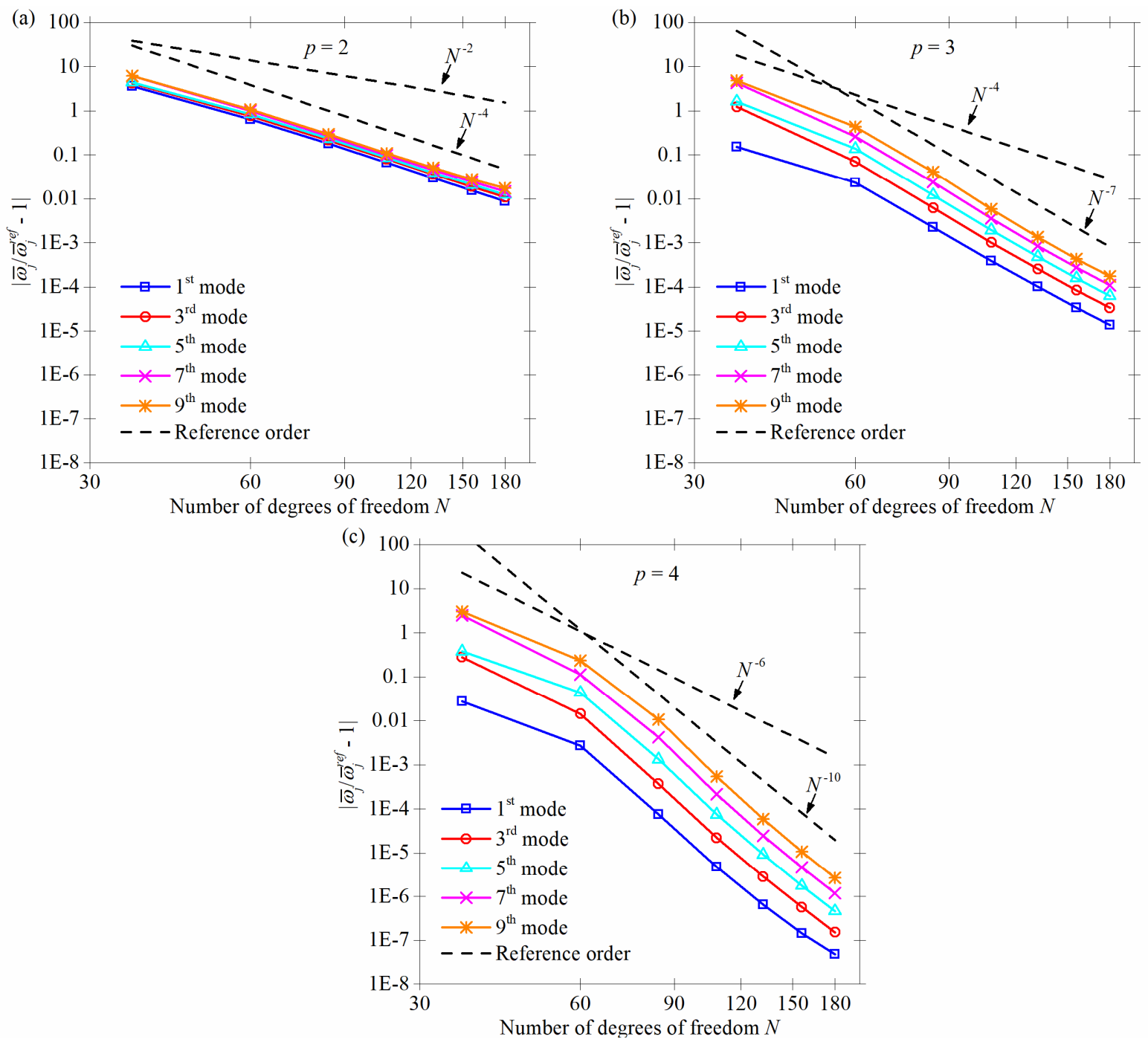

Fig. 15. Circular ring - Convergence test for different NURBS degree $p$. (a) $p=2$. (b) $p=3$. (c) $p=$ 4.

The normalized numeric discrete spectra are also investigated for this full ring, and we conduct the analysis for both the $2 \mathrm{nEB}$ element and the developed formulation. The cubic NURBS curves are used. Three different values of the number of DOFs $N$ are considered, i.e., $N=708,1188$, and 1668 . The computed spectra are depicted in Fig. 16a-b. The same conclusion as stated in the previous example is obtained. The present approach offers better performance and accuracy. Up to $50 \%$ of frequencies, obtained by the proposed approach, and approximately $40 \%$ of frequencies, obtained by the $2 \mathrm{nEB}$ element, have to the relative error less than $2.5 \%$.

The degree $p$, i.e., $p=2,3$, and 4 , of the NURBS curves alters the normalized numeric discrete spectra. As shown in Fig. 17, significant improvement in the accuracy of high frequencies can be reached by using 

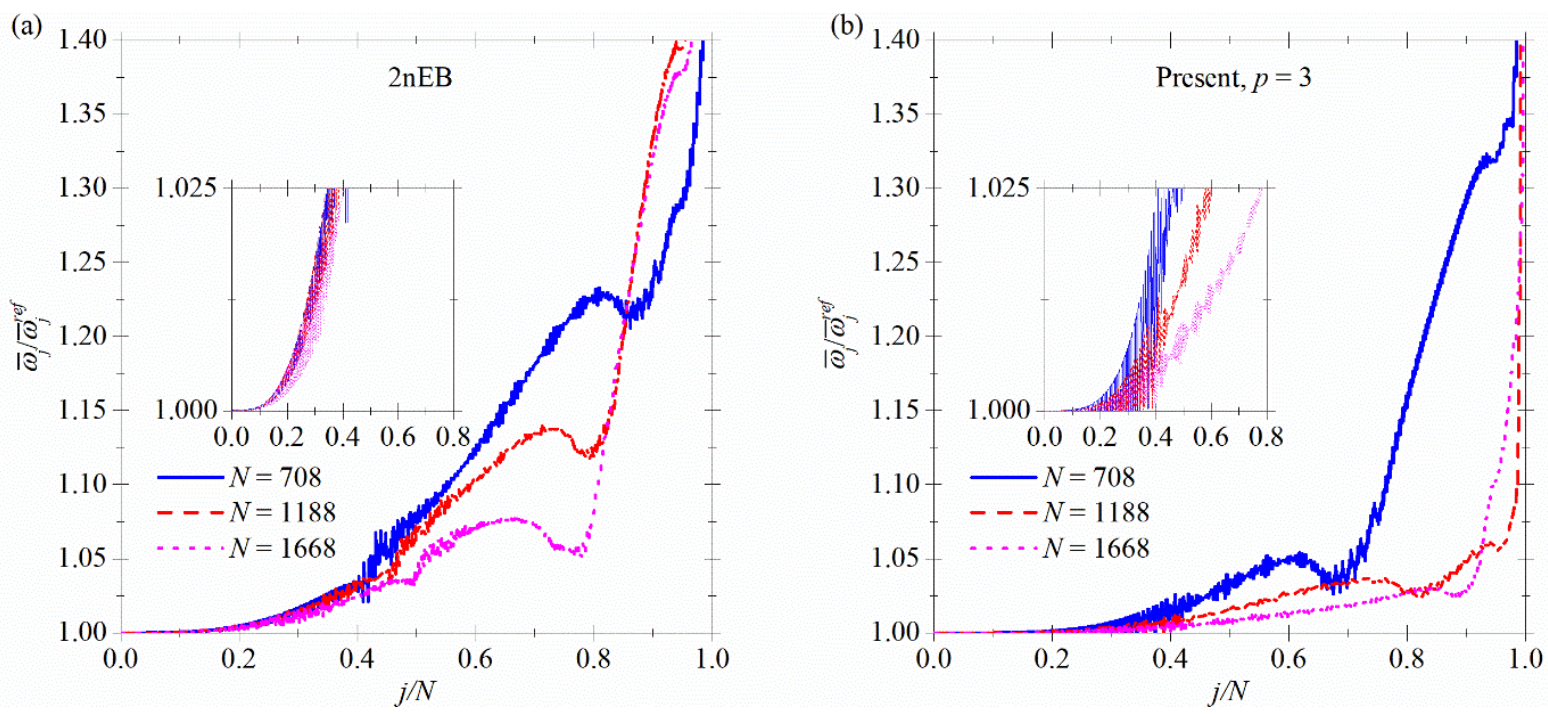

Fig. 16. Circular ring - Normalized numeric discrete spectra. (a) the 2nEB formulation. (b) The proposed formulation.

higher degrees of the NURBS curves. In analogous to the previous example, with a specific value of $N$, the outlier phenomenon appears at the same value of $j / N$ regardless of the considered NURBS degree $p$.

\subsection{An L-shaped frame}

This example aims to validate the capacity of the proposed formulation for analysis of beam structures with kinks. An L-shaped frame with a kink shown in Fig. 18a is thus considered. The L-shaped frame consists of two segments, i.e., $B C$ and $C D$, with a right angle at $C$. The frame is pinned at $B$ and $D$. The two segments are in the same length of $L$. For the use of rotation-free isogeometric beam formulations, the rigid connection at $C$ can be treated by a bending strip as shown in Fig. 18b. As already stated, the proposed formulation does not require any special treatment for the connection.

A natural frequency of the frame is normalized through

$$
\bar{\omega}_{j}=\omega_{j} L^{2} \sqrt{\frac{\rho A}{E I}} .
$$

The first five $\bar{\omega}_{j}$ obtained by the $2 \mathrm{nEB}$ element and the present approach, and their mode shapes are given in Table 3 and Fig. 19. Very good agreement is obtained as the difference on the frequency and mode shapes is small. 

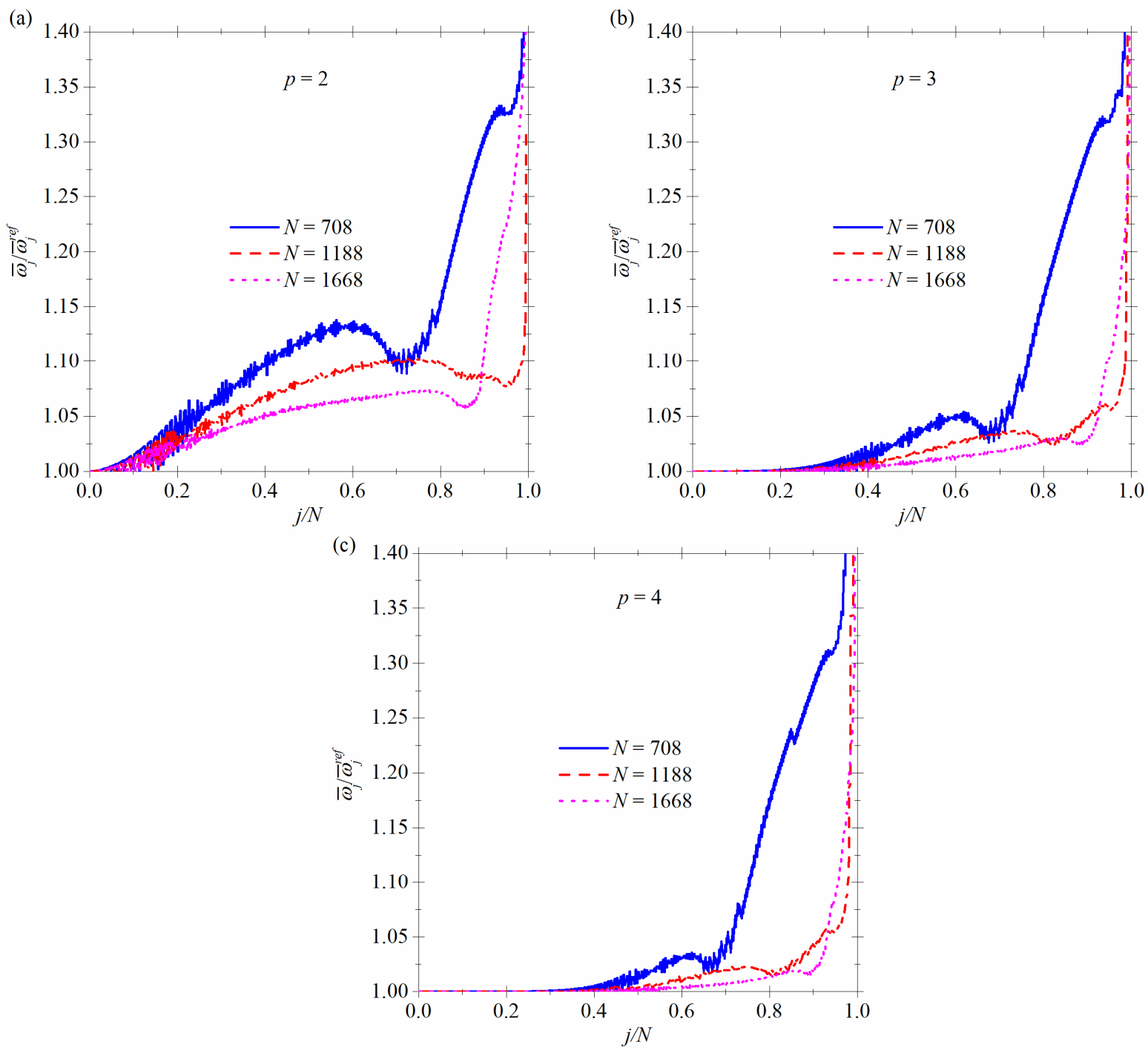

Fig. 17. Circular ring - Normalized numeric discrete spectra with different NURBS degree $p$. (a) $p=$ 2. (b) $p=3$. (c) $p=4$.

We observe from the mode shapes that, compared to the transverse displacement, the axial displacement is small. In other words, vibration analysis of the frame can be characterized by the transverse displacement. With the 2nEB element, the transverse displacement is approximated by using a cubic polynomial, achieving $C^{1}$ continuity between elements. In the proposed approach, the NURBS basis functions are used for approximations of both the geometry and displacements. However, since the geometry of the frame consists of only two straight segments, the NURBS basis functions are thus polynomials. Furthermore, the continuity between elements can be easily adjusted, and it is possible to attain higher continuity than $C^{1}$.

The effect of inter-element continuity on the order of convergence is examined. The convergence in terms of relative errors for the first five $\bar{\omega}_{j}$ is studied. Cubic NURBS curves with $C^{1}$ and $C^{2}$ continuity are 
(a)

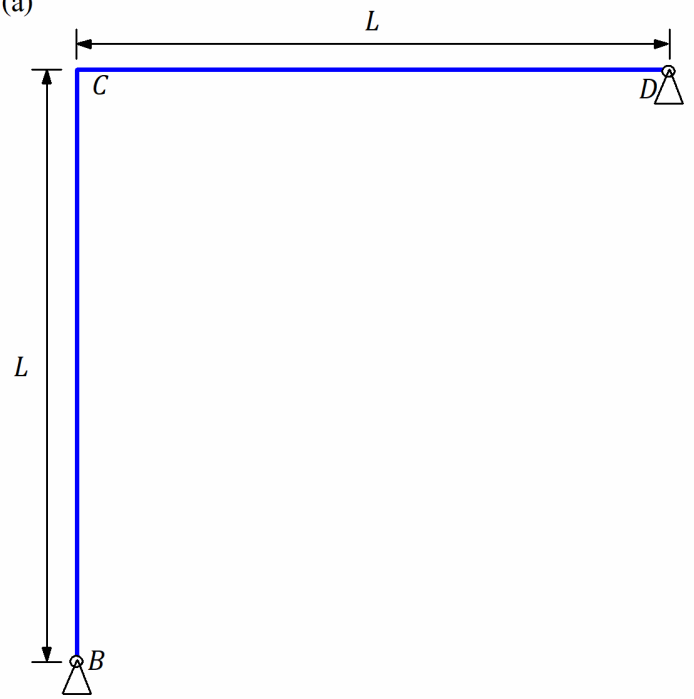

(b)

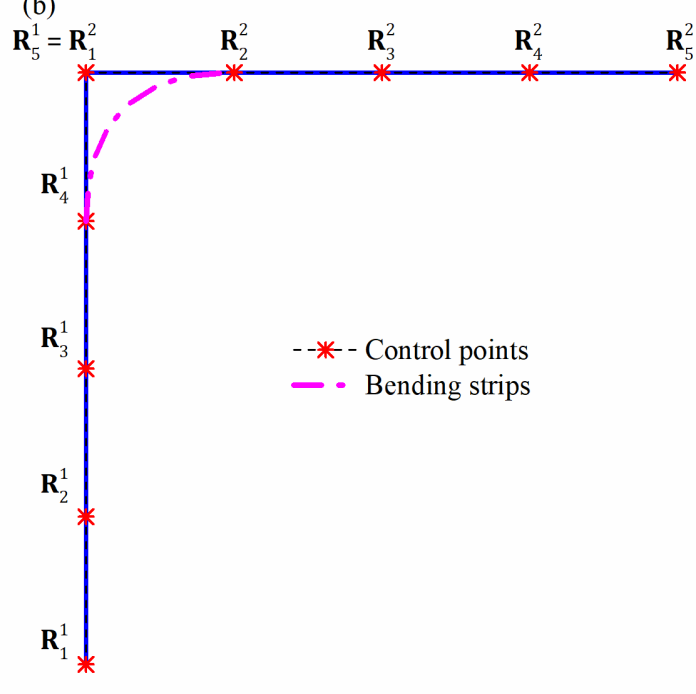

Fig. 18. L-shaped frame. (a) Dimensions and boundary conditions. (b) Bending strips for the enforcement of the rigid connections.

Table 3. L-shaped frame. Comparison of the first five normalized natural frequencies $\bar{\omega}_{j}=$

$$
\omega_{j} L^{2} \sqrt{\rho A / E I} .
$$

\begin{tabular}{lll}
\hline Mode & 2nEB & Present \\
\hline 1 & 9.868 & 9.868 \\
2 & 15.413 & 15.413 \\
3 & 39.459 & 39.459 \\
4 & 49.914 & 49.914 \\
5 & 88.726 & 88.726 \\
\hline
\end{tabular}

used for comparison with the 2nEB element. Convergence results are plotted in Fig. 20a for the 2nEB element, and in Fig. 20b-c for our approach with $C^{1}$ and $C^{2}$ continuity, respectively. In this example, it is found that the order of convergence produced by the $2 \mathrm{nEB}$ element is higher than that in the previous two examples, i.e., 4 and 2. This behavior can be expected due to the simple initial configuration with straight lines. On the other hand, the order of convergence obtained by the present work is affected by the interelement continuity, i.e., the orders of convergence with $C^{1}$ and $C^{2}$ continuity are, respectively, 4 and 
(a)

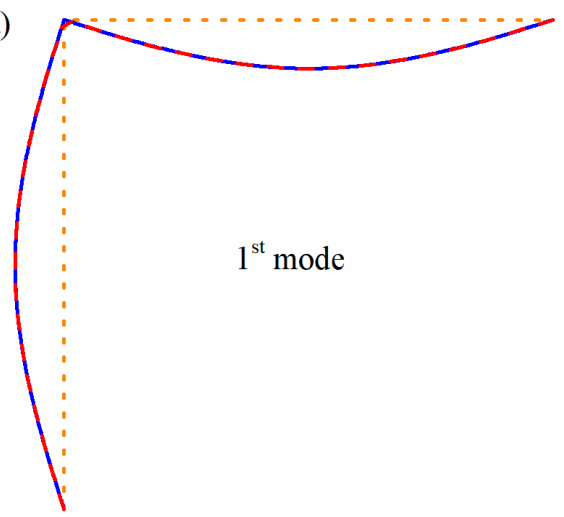

(c)

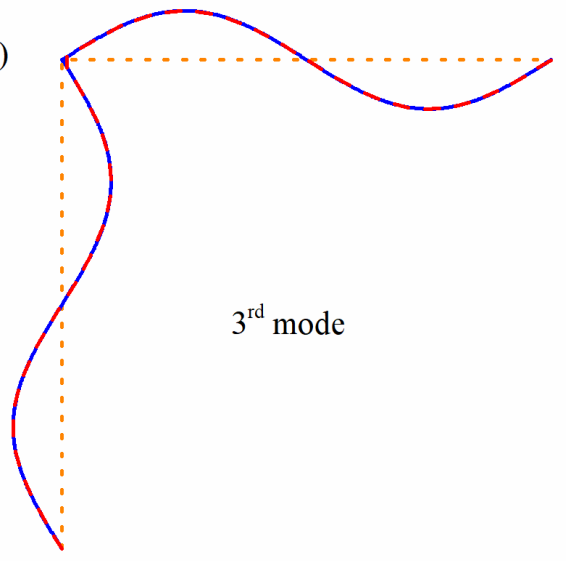

(b)

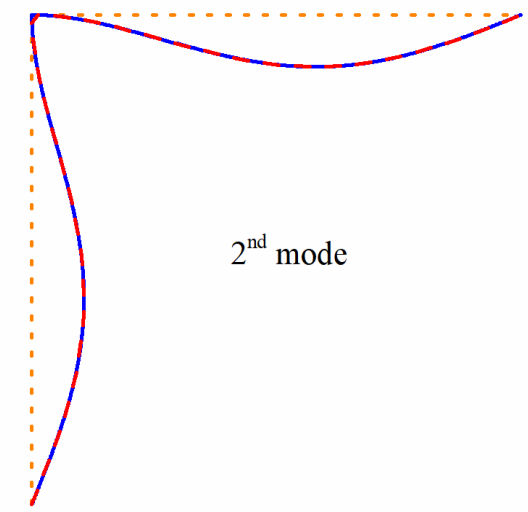

(d)

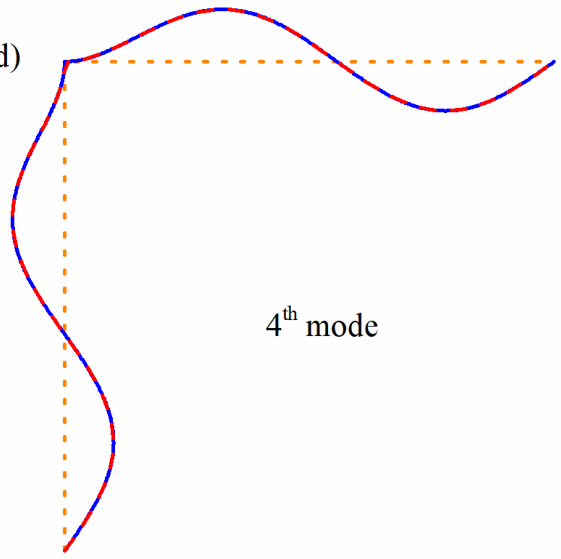

(e)

Fig. 19. L-shaped frame - First five mode shapes.

5. The improvement of the order of convergence with higher inter-element continuity agrees well with the observation reported in [26].

The relative errors for the first five $\bar{\omega}_{j}$ are plotted in Fig. 21a-c for quadratic, cubic, and quartic NURBS curves, respectively. It is found that $3 p-4$ is the order of convergence. For the quartic NURBS curves, the relative errors of the first and second frequencies are dramatically decreased to values between $10^{-9}$ and $10^{-8}$, and then remain unchanged regardless of the increase of DOFs. 

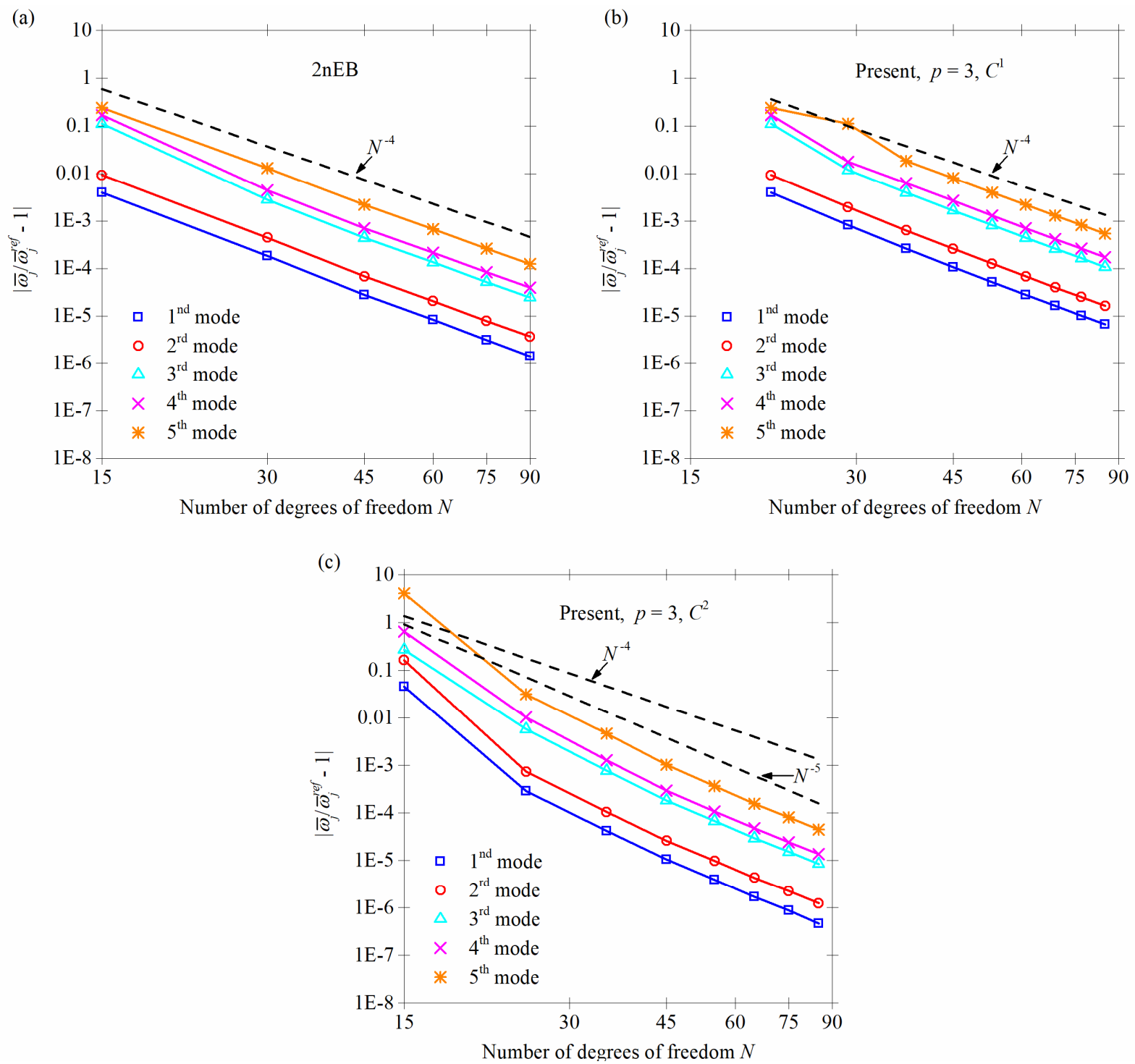

Fig. 20. L-shaped frame - Convergence test for first five $\bar{\omega}_{j}$ between the $2 \mathrm{nEB}$ and the proposed formulation with cubic NURBS curves.

\subsection{Lattice tower}

The purpose of the last example is to show the applicability of the proposed formulation in modeling complicated beam structures. We thus consider a tower-like lattice structure portrayed in Fig. 22. The lattice tower consists of 18 segments differently oriented at several joints. Each segment can be represented by a quadratic NURBS curve with the locations of control points given in Appendix. 
(a)

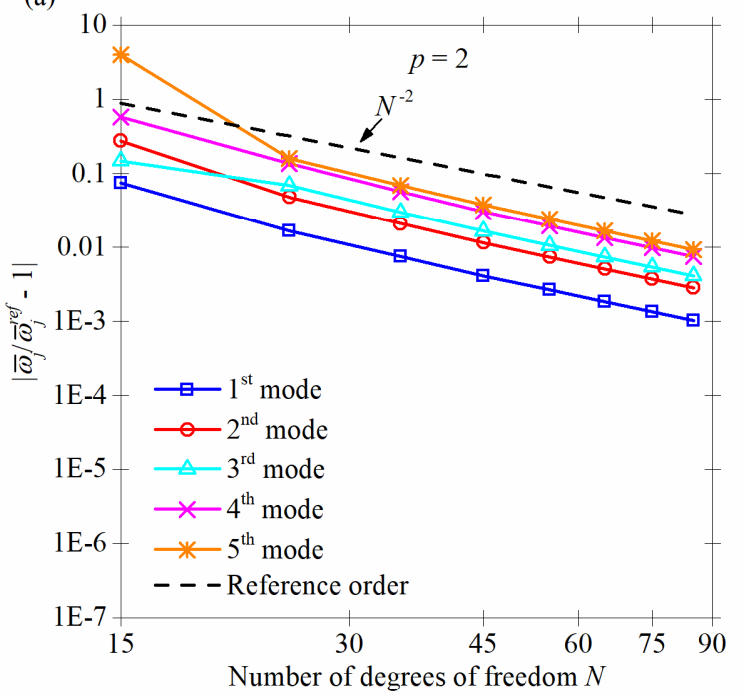

(b)

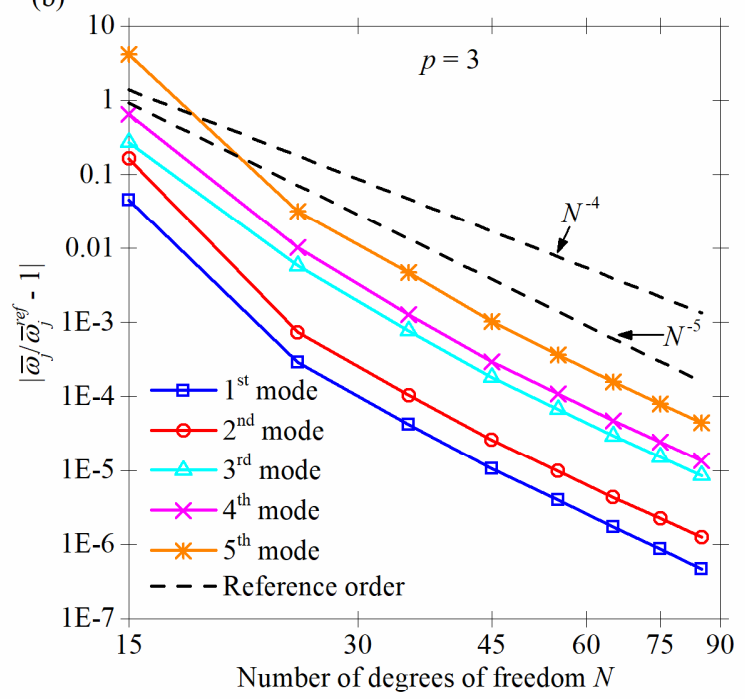

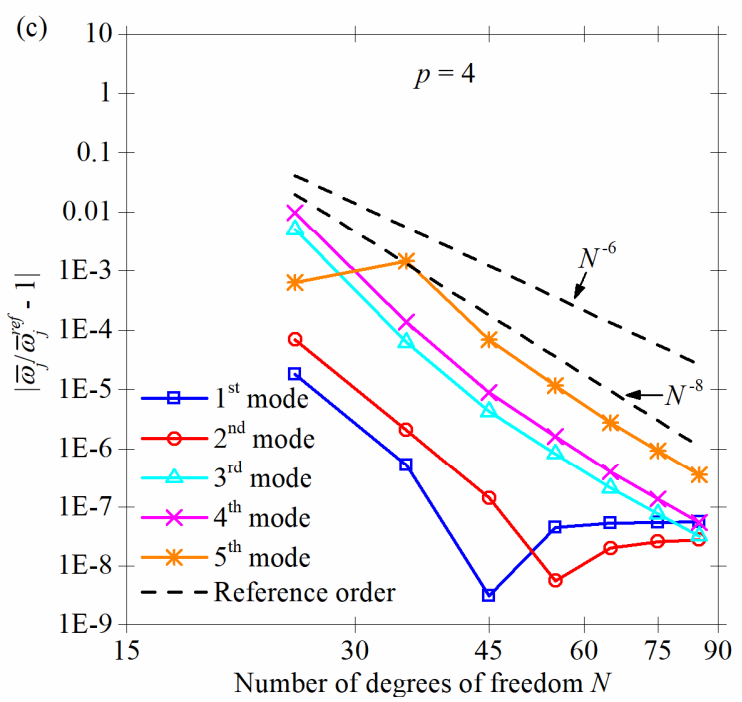

Fig. 21. L-shaped frame - Convergence test for different NURBS degree $p$. (a) $p=2$. (b) $p=3$. (c)

$$
p=4 \text {. }
$$

The first five frequencies $\omega_{j}$ computed with the $2 \mathrm{nEB}$ element and the present approach are listed in Table 4. Apparently, the two solutions are in good agreement. The first five mode shapes are also plotted in Fig. 23, and the difference between two approaches is indistinguishable.

Fig. 24 shows a comparison of the relative errors for the first five $\omega_{j}$ between the $2 \mathrm{nEB}$ element and the present approach. Here, cubic NURBS curves are used for the comparison. It can be stated that the order of convergence offered by the $2 \mathrm{nEB}$ element is reduced for beam structures with complex initial configurations. The developed formulation, however, yields better performance and faster convergence than those by the 2 nEB element. 


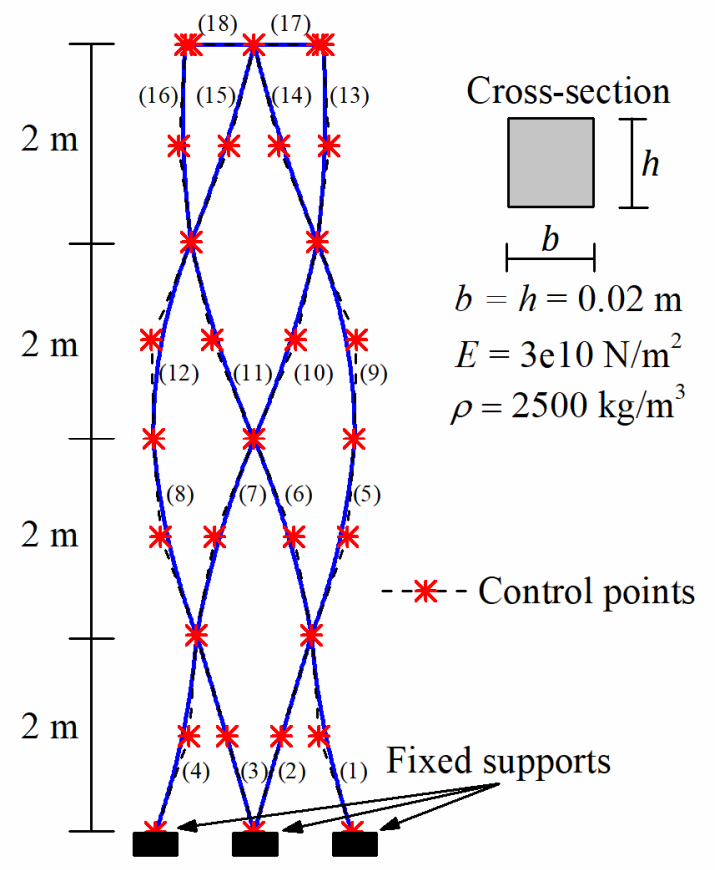

Fig. 22. Lattice tower - Problem setup.

Table 4. Lattice tower. Comparison of the first five natural frequencies $\omega_{j}$.

\begin{tabular}{lll}
\hline Mode & 2nEB & Present \\
\hline 1 & 7.137 & 7.136 \\
2 & 16.372 & 16.371 \\
3 & 42.383 & 42.376 \\
4 & 52.341 & 52.336 \\
5 & 63.460 & 63.460 \\
\hline
\end{tabular}

\section{Extensibility to analysis of spatial beams}

In practice, planar beam structures whose beam axes are laid in a same plane are frequently encountered, e.g., portal frames and multistorey building with bracing systems. The analysis of these beam structures can be apparently carried out by utilizing the spatial beam formulations. However, in the case the cross-section is doubly symmetric or the cross-section height is small in comparison to the radius of 


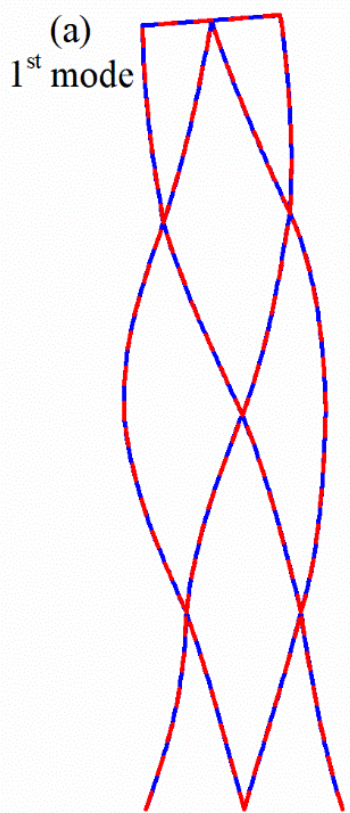

(d) $4^{\text {th }}$ mode

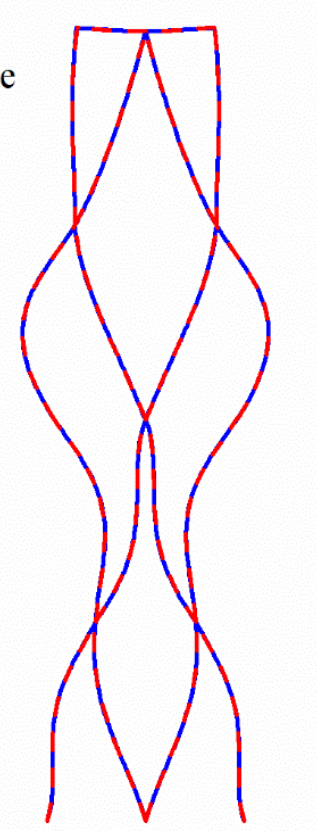

(b) $2^{\text {nd }}$ mode

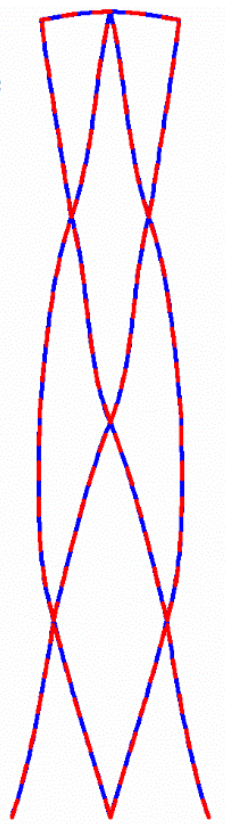

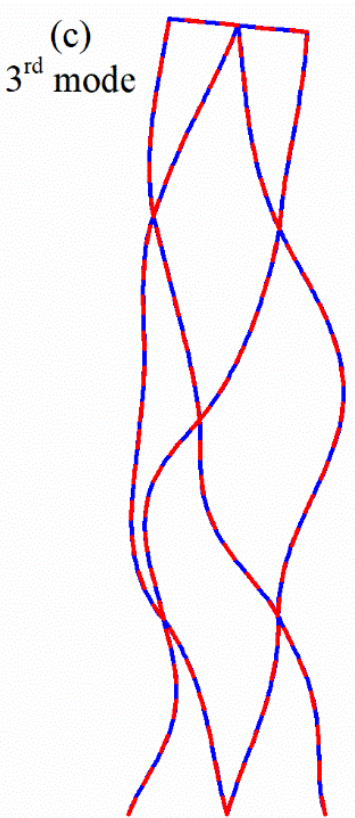

(e)

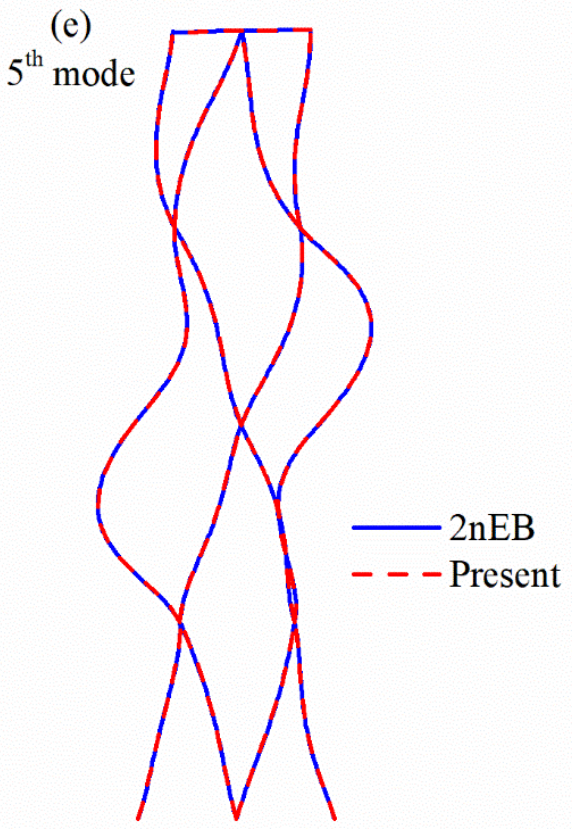

Fig. 23. Lattice tower - First five mode shapes.

curvature of the beam axis, in-plane and out-of-plane vibrations are decoupled [18, 30, 31]. In other words, the in-plane vibration of these beam structures can be performed independently with the out-of-plane vibration. This feature facilitates the utilization of the planar Euler-Bernoulli beam formulations in many scenarios, e.g., conceptual designs. In fact, in these circumstances, the planar beam formulations including the one proposed in this study offer more efficient performances owing to reductions in computational 


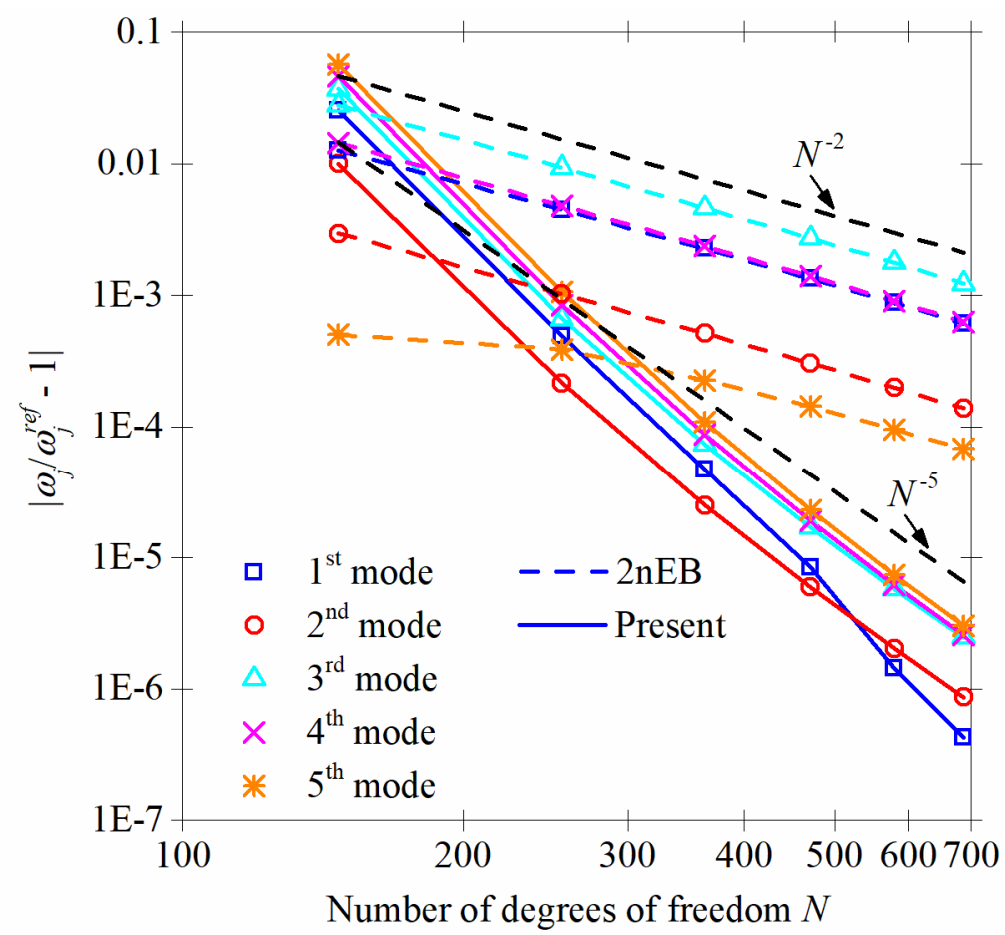

Fig. 24. Lattice tower - Convergence test for the first five $\omega_{j}$ between the $2 \mathrm{nEB}$ element and the proposed formulation with cubic NURBS curves.

costs. As a result, there is a huge body of works dedicated to the development of planar beam formulations for the in-plane vibration of planar beam structures, and research in this field is still progressing.

Nevertheless, the use of spatial beam formulations is inevitable to have full assessments of behaviors of beam structures. Regarding isogeometric analysis of spatial Euler-Bernoulli beams, displacements of the beam axis and axial rotation of the cross-sections are commonly considered as unknown kinematics for the description of beams, e.g., see [32-35]. As a result, due to the absence of total cross-sectional rotation, these beam formulations exhibit the analogous drawbacks with planar rotation-free counterparts, i.e., rigid multipatch connections and prescriptions of rotational boundary conditions. To remove these drawbacks, the approach in this study can be generalized, i.e., incorporating the total cross-sectional rotations at the ends of the patch as discrete unknowns. This method has been implemented in [36] for linear analysis and in [37] for geometrically nonlinear analysis. However, applications in dynamic analysis are still not yet examined. In the following, the extensibility of the method presented in section 2.5 is briefly discussed for the analysis of spatial beams, and detailed derivations are reserved for a follow-up paper.

For descriptions of spatial Euler-Bernoulli beams, the unknown kinematics, i.e., displacements of the beam axis $\mathbf{u}_{0}$ and axial rotation of the cross-section $\phi$, are discretized as 


$$
\mathbf{u}_{0}(\xi, t)=\sum_{i=1}^{n} R_{i}(\xi) \mathbf{u}_{i}(t) \quad \phi(\xi, t)=\sum_{i=1}^{n} R_{i}(\xi) \phi_{i}(t) .
$$

In the sense of small displacements and rotations, relations between the displacements of the second and second-to-last control points (i.e., $\mathbf{R}_{2}$ and $\left.\mathbf{R}_{n-1}\right)$ and the end total cross-sectional rotations ( $\boldsymbol{\phi}_{1}$ and $\left.\boldsymbol{\phi}_{n}\right)$ of a patch can be derived as

$$
\begin{aligned}
& \mathbf{u}_{2}(t)=\mathbf{u}_{1}(t)-L_{1} \mathbf{T}_{1} \times \boldsymbol{\phi}_{1}+\Delta L_{1} \mathbf{T}_{1} \\
& \mathbf{u}_{n-1}(t)=\mathbf{u}_{n}(t)+L_{n-1} \mathbf{T}_{n} \times \boldsymbol{\phi}_{n}-\Delta L_{n-1} \mathbf{T}_{n} .
\end{aligned}
$$

One can notice the analogy of expressions in Eqs. (50) and (51) with those in Eqs. (30) and (31), i.e., the end rotations $\theta_{1}$ and $\theta_{n}$ are, respectively, replaced by the end total cross-sectional rotations $\boldsymbol{\phi}_{1}$ and $\boldsymbol{\phi}_{n}$. Furthermore, the relations between axial rotations $\left(\phi_{1}\right.$ and $\left.\phi_{n}\right)$ and total cross-sectional rotations ( $\boldsymbol{\phi}_{1}$ and $\left.\boldsymbol{\phi}_{n}\right)$ at the ends of the patch can be established as

$$
\phi_{1}=\phi_{1} \cdot \mathbf{T}_{1}=\boldsymbol{\phi}_{n} \cdot \mathbf{T}_{n}
$$

Eventually, the dependency of $\mathbf{u}_{2}, \mathbf{u}_{n-1}, \phi_{1}$, and $\phi_{n}$ on $\boldsymbol{\phi}_{1}$ and $\boldsymbol{\phi}_{n}$ as shown in Eqs. (50)-(52) allows the consideration of the total cross-sectional rotations at the ends of the patch as discrete unknowns. The accuracy and efficiency of this approach will be thoroughly manifested in a forthcoming paper.

\section{Conclusions}

In this paper, we have developed a novel beam formulation for dynamic analysis of multi-patch planar Euler-Bernoulli beams. In this setting, only the translational displacements of the beam axis are used for kinematic descriptions. NURBS curves are used for approximations of the beam axis and its displacements. At the two ends of the beam, cross-sectional rotations are used as DOFs by a transformation between these rotations and control variables. The present formulation not only preserves the advantages but also eliminates various shortcomings of the existing rotation-free isogeometric beam formulations, i.e., simple modeling of beams with rigid multi-patch connections and straightforward applications of rotational boundary conditions.

The accuracy of the proposed formulation is numerically confirmed as the computed results are compared with those derived from the conventional two-noded Euler-Bernoulli finite beam element $(2 \mathrm{nEB})$. In all numerical examples, the proposed formulation offers better convergence and higher accuracy compared to the $2 \mathrm{nEB}$ element. An improvement in the accuracy of frequencies by using NURBS curves 
of high degree is observed. The proposed formulation converges with higher order than $2(p-1)$, which is reported in the literature. In addition, the beams considered in the examples represent many types of practical beam structures, i.e., curved beams with circular shapes, frames with kinks, lattice structures with arbitrarily oriented segments. With these features, the accuracy and efficiency of the proposed formulation are fully validated.

Lastly, the extensibility of the proposed formulation to the case of spatial beams is briefly discussed with a short introduction to the dependency of displacements of the second and second-to-last control points, and also the axial rotations on total cross-sectional rotations at the ends of a patch. This approach will be thoroughly presented and elaborated in a forthcoming paper.

\section{Acknowledgements}

Scholarships from the ASEAN University Network/Southeast Asia Engineering Education Development Network (AUN/SEED-Net) and Sirindhorn International Institute of Technology (SIIT) for the first author are greatly appreciated.

\section{Appendix. Control points of the lattice tower}

\begin{tabular}{cccc}
\hline Patch & $1^{\text {st }}$ control point & $2^{\text {nd }}$ control point & $3^{\text {rd }}$ control point \\
\hline 1 & $(1,0)$ & $(0.662,1)$ & $(0.583,2)$ \\
3 & $(0,0)$ & $(0.276,1)$ & $(0.583,2)$ \\
4 & $(0,0)$ & $(-0.276,1)$ & $(-0.583,2)$ \\
5 & $(-1,0)$ & $(-0.662,1)$ & $(-0.583,2)$ \\
6 & $(0.583,2)$ & $(0.404,3)$ & $(1.022,4)$ \\
7 & $(-0.583,2)$ & $(-0.404,3)$ & $(0,4)$ \\
8 & $(-0.583,2)$ & $(-0.953,3)$ & $(-1.022,4)$ \\
9 & $(1.022,4)$ & $(1.040,5)$ & $(0.640,6)$
\end{tabular}




\begin{tabular}{cccc}
10 & $(0,4)$ & $(0.423,5)$ & $(0.640,6)$ \\
11 & $(0,4)$ & $(-0.424,5)$ & $(-0.640,6)$ \\
12 & $(-1.022,4)$ & $(-1.040,5)$ & $(-0.640,6)$ \\
13 & $(0.640,6)$ & $(0.764,7)$ & $(0.700,8)$ \\
14 & $(0.640,6)$ & $(0.255,7)$ & $(0,8)$ \\
15 & $(-0.640,6)$ & $(-0.255,7)$ & $(0,8)$ \\
16 & $(-0.640,6)$ & $(-0.764,7)$ & $(-0.700,8)$ \\
17 & $(0.700,8)$ & $(0.640,8)$ & $(0,8)$ \\
18 & $(0,8)$ & $(-0.640,8)$ & $(-0.700,8)$ \\
\hline
\end{tabular}

\section{References}

[1] J.A. Cottrell, A. Reali, Y. Bazilevs, T.J.R. Hughes, Isogeometric analysis of structural vibrations, Computer Methods in Applied Mechanics and Engineering, 195 (41) (2006) 5257-5296.

[2] A. Reali, An isogeometric analysis approach for the study of structural vibrations, Journal of Earthquake Engineering, 10 (1) (2006) 1-30.

[3] J. Kiendl, K.U. Bletzinger, J. Linhard, R. Wüchner, Isogeometric shell analysis with Kirchhoff-Love elements, Computer Methods in Applied Mechanics and Engineering, 198 (49-52) (2009) 3902-3914.

[4] J. Kiendl, Y. Bazilevs, M.C. Hsu, R. Wüchner, K.U. Bletzinger, The bending strip method for isogeometric analysis of Kirchhoff-Love shell structures comprised of multiple patches, Computer Methods in Applied Mechanics and Engineering, 199 (37-40) (2010) 2403-2416.

[5] W. Dornisch, R. Müller, S. Klinkel, An efficient and robust rotational formulation for isogeometric Reissner-Mindlin shell elements, Computer Methods in Applied Mechanics and Engineering, 303 (2016) 1-34.

[6] T. Yu, J. Zhang, H. Hu, T.Q. Bui, A novel size-dependent quasi-3D isogeometric beam model for twodirectional FG microbeams analysis, Composite Structures, 211 (2019) 76-88.

[7] D. Vo, P. Nanakorn, A total Lagrangian Timoshenko beam formulation for geometrically nonlinear isogeometric analysis of planar curved beams, Acta Mechanica, (2020). 
[8] D. Vo, P. Nanakorn, T.Q. Bui, A total Lagrangian Timoshenko beam formulation for geometrically nonlinear isogeometric analysis of spatial beam structures, Acta Mechanica, 231 (9) (2020) 3673-3701.

[9] T.Q. Bui, Extended isogeometric dynamic and static fracture analysis for cracks in piezoelectric materials using NURBS, Computer Methods in Applied Mechanics and Engineering, 295 (2015) 470-509. [10] J. Kiendl, M. Ambati, L. De Lorenzis, H. Gomez, A. Reali, Phase-field description of brittle fracture in plates and shells, Computer Methods in Applied Mechanics and Engineering, 312 (2016) 374-394.

[11] C. Wang, T. Yu, J.L. Curiel-Sosa, N. Xie, T.Q. Bui, Adaptive chaotic particle swarm algorithm for isogeometric multi-objective size optimization of FG plates, Structural and Multidisciplinary Optimization, 60 (2) (2019) 757-778.

[12] O. Weeger, B. Narayanan, M.L. Dunn, Isogeometric shape optimization of nonlinear, curved 3D beams and beam structures, Computer Methods in Applied Mechanics and Engineering, 345 (2019) 26-51.

[13] S.J. Lee, K.S. Park, Vibrations of Timoshenko beams with isogeometric approach, Applied Mathematical Modelling, 37 (22) (2013) 9174-9190.

[14] A.-T. Luu, N.-I. Kim, J. Lee, Isogeometric vibration analysis of free-form Timoshenko curved beams, Meccanica, 50 (1) (2015) 169-187.

[15] A.-T. Luu, N.-I. Kim, J. Lee, NURBS-based isogeometric vibration analysis of generally laminated deep curved beams with variable curvature, Composite Structures, 119 (2015) 150-165.

[16] T.-A. Huynh, A.-T. Luu, J. Lee, Bending, buckling and free vibration analyses of functionally graded curved beams with variable curvatures using isogeometric approach, Meccanica, 52 (11) (2017) 2527-2546. [17] X. Zhang, Y. Xia, Q. Hu, P. Hu, Efficient isogeometric formulation for vibration analysis of complex spatial beam structures, European Journal of Mechanics - A/Solids, 66 (2017) 212-231.

[18] H. Liu, X. Zhu, D. Yang, Isogeometric method based in-plane and out-of-plane free vibration analysis for Timoshenko curved beams, Structural Engineering and Mechanics, 59 (3) (2016) 503-526.

[19] A.P. Nagy, M.M. Abdalla, Z. Gürdal, Isogeometric design of elastic arches for maximum fundamental frequency, Structural and Multidisciplinary Optimization, 43 (1) (2011) 135-149.

[20] O. Weeger, U. Wever, B. Simeon, Isogeometric analysis of nonlinear Euler-Bernoulli beam vibrations, Nonlinear Dynamics, 72 (4) (2013) 813-835.

[21] D. Wang, W. Liu, H. Zhang, Superconvergent isogeometric free vibration analysis of Euler-Bernoulli beams and Kirchhoff plates with new higher order mass matrices, Computer Methods in Applied Mechanics and Engineering, 286 (2015) 230-267.

[22] F. Maurin, L. Dedè, A. Spadoni, Isogeometric rotation-free analysis of planar extensible-elastica for static and dynamic applications, Nonlinear Dynamics, 81 (1) (2015) 77-96. 
[23] H. Askari, E. Esmailzadeh, A. Barari, A unified approach for nonlinear vibration analysis of curved structures using non-uniform rational b-spline representation, Journal of Sound and Vibration, 353 (2015) 292-307.

[24] V.N. Van Do, T.H. Ong, C.H. Thai, Dynamic responses of Euler-Bernoulli beam subjected to moving vehicles using isogeometric approach, Applied Mathematical Modelling, 51 (2017) 405-428.

[25] S.F. Hosseini, A. Hashemian, A. Reali, On the application of curve reparameterization in isogeometric vibration analysis of free-from curved beams, Computers \& Structures, 209 (2018) 117-129.

[26] A. Borković, S. Kovačević, G. Radenković, S. Milovanović, D. Majstorović, Rotation-free isogeometric dynamic analysis of an arbitrarily curved plane Bernoulli-Euler beam, Engineering Structures, 181 (2019) 192-215.

[27] S.B. Raknes, X. Deng, Y. Bazilevs, D.J. Benson, K.M. Mathisen, T. Kvamsdal, Isogeometric rotationfree bending-stabilized cables: Statics, dynamics, bending strips and coupling with shells, Computer Methods in Applied Mechanics and Engineering, 263 (2013) 127-143.

[28] D. Vo, P. Nanakorn, Geometrically nonlinear multi-patch isogeometric analysis of planar curved Euler-Bernoulli beams, Computer Methods in Applied Mechanics and Engineering, 366 (2020) 113078.

[29] L. Piegl, W. Tiller, The NURBS book, 2nd ed., Springer, New York, 1997.

[30] S.Y. Lee, J.C. Chao, Exact solutions for out-of-plane vibration of curved nonuniform beams, Journal of Applied Mechanics, 68 (2) (2000) 186-191.

[31] S.Y. Lee, J.Y. Hsiao, Free in-plane vibrations of curved nonuniform beams, Acta Mechanica, 155 (3) (2002) 173-189.

[32] L. Greco, M. Cuomo, B-spline interpolation of Kirchhoff-Love space rods, Computer Methods in Applied Mechanics and Engineering, 256 (2013) 251-269.

[33] G. Radenković, A. Borković, Linear static isogeometric analysis of an arbitrarily curved spatial Bernoulli-Euler beam, Computer Methods in Applied Mechanics and Engineering, 341 (2018) 360-396.

[34] A.M. Bauer, M. Breitenberger, B. Philipp, R. Wüchner, K.U. Bletzinger, Nonlinear isogeometric spatial Bernoulli beam, Computer Methods in Applied Mechanics and Engineering, 303 (2016) 101-127.

[35] A.M. Bauer, R. Wüchner, K.U. Bletzinger, Weak coupling of nonlinear isogeometric spatial Bernoulli beams, Computer Methods in Applied Mechanics and Engineering, (2019) 112747.

[36] L. Greco, M. Cuomo, An implicit g1 multi patch b-spline interpolation for Kirchhoff-Love space rod, Computer Methods in Applied Mechanics and Engineering, 269 (2014) 173-197.

[37] D. Vo, P. Nanakorn, T.Q. Bui, Geometrically nonlinear multi-patch isogeometric analysis of spatial Euler-Bernoulli beams, Computer Methods in Applied Mechanics and Engineering, -- (--) (2020) --_--. (submitted, under review) 